Electronic Supplementary Information

\title{
Highly Stable 1,2-Dicarbonyl Radical Cations Derived from N-Heterocyclic Carbenes
}

\author{
Youngsuk Kim, ${ }^{\mathrm{a}}$ Jung Eun Byeon, ${ }^{\mathrm{a}} \mathrm{Gu}$ Yoon Jeong, ${ }^{\mathrm{a}}$ Seoung Su Kim, ${ }^{\mathrm{a}}$ \\ Hayoung Song, ${ }^{a}$ Eunsung Lee*,a \\ ${ }^{a}$ Department of Chemistry, Pohang University of Science and Technology (POSTECH), \\ Pohang, 37673, Republic of Korea \\ *E-mail: eslee@postech.ac.kr
}




\section{Table of contents}

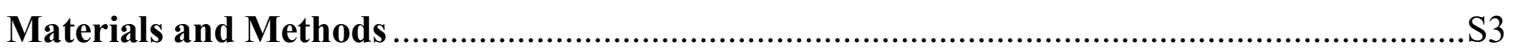

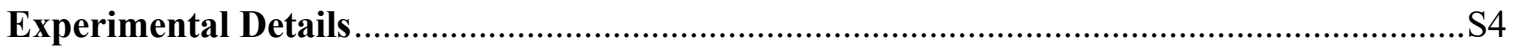

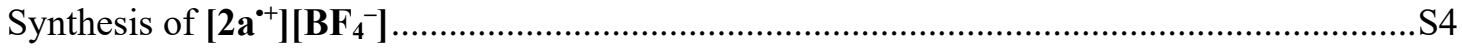

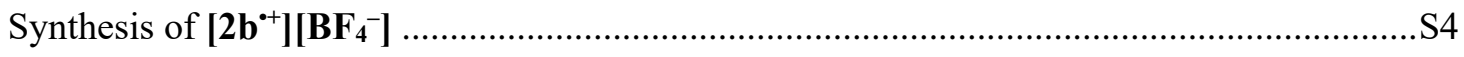

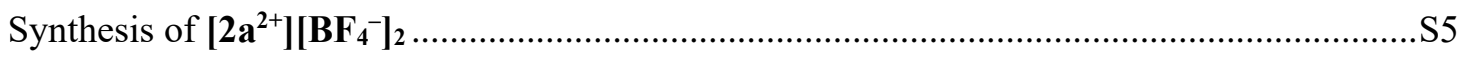

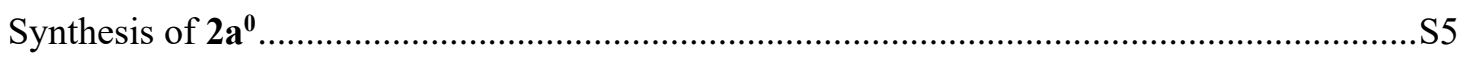

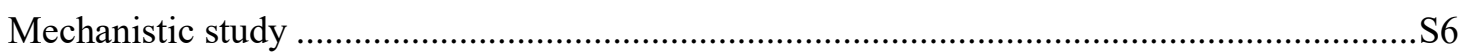

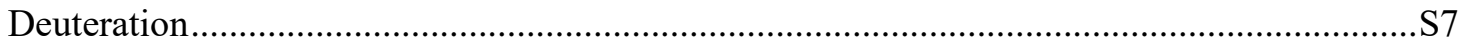

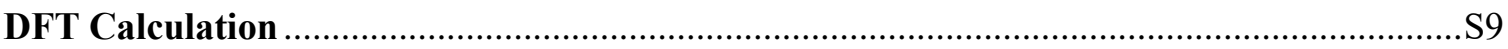

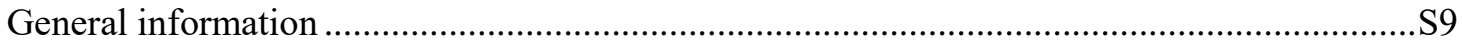

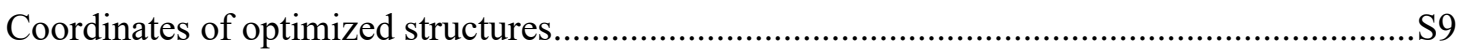

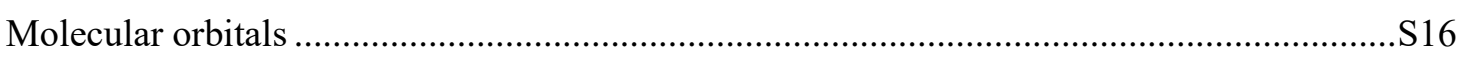

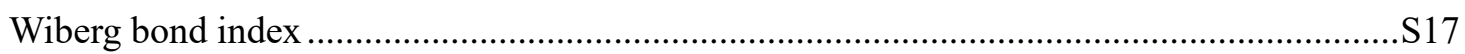

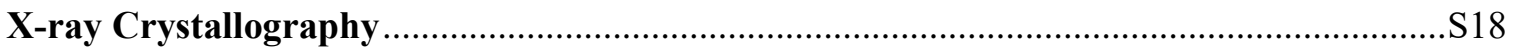

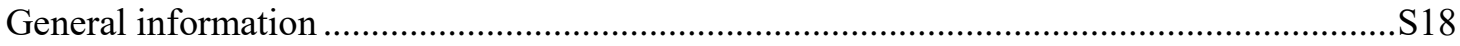

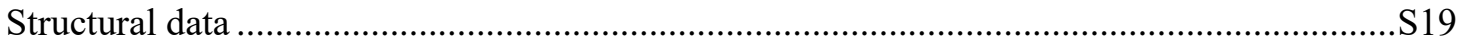

EPR

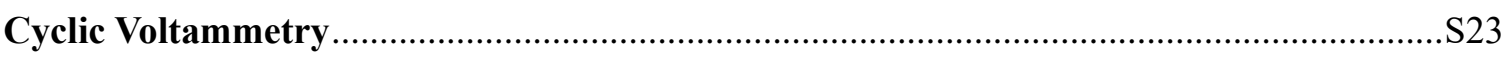

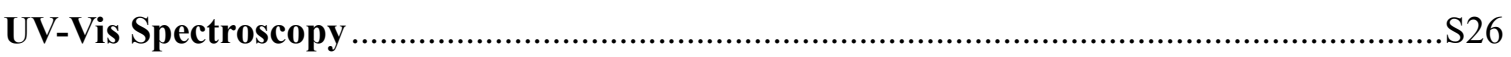

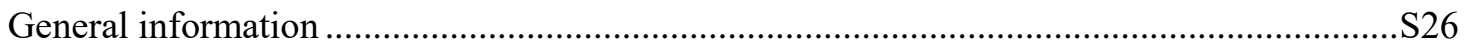

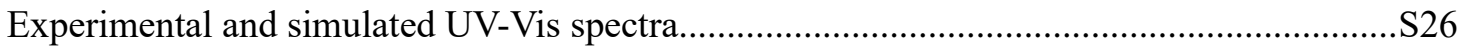

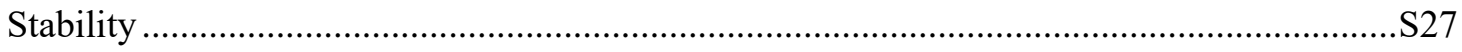

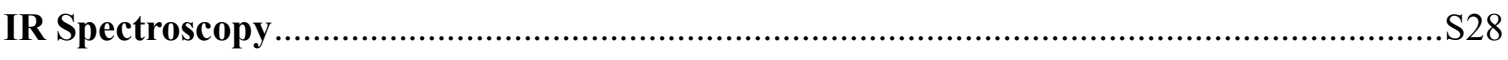

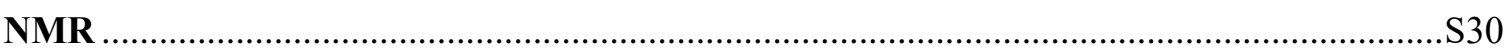

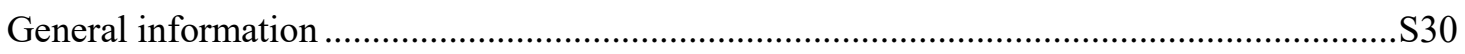

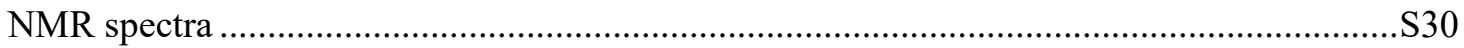

References 


\section{Materials and Methods}

\section{General methods}

All air- and moisture-insensitive reactions were carried out under an ambient atmosphere, magnetically stirred. All air- and moisture-sensitive manipulations were performed using ovendried glassware, including standard Schlenk and glovebox techniques under an atmosphere of nitrogen. High resolution mass spectrometry was performed at the Korea Basic Science Institute with a JEOL JMS 700 high resolution mass spectrometer. Thermal gravimetric analyses (TGA) were performed under air with a Perkin Elmer Pyris 1. Elemental analyses were performed at Ulsan National Institute of Science and Technology (UNIST) Central Research Facilities with a Leco Truspec Micro elemental analyzer. ESI-MS spectrometry was performed with a Thermofisher LTQ XL Mass Spectrometer.

\section{Reagents}

IMes (1a), IDipp (1b), and $\mathrm{IMesH}^{+} \mathrm{Cl}^{-}$were synthesized according to a reference. ${ }^{[1]}$ Oxalyl chloride $(\mathrm{COCl})_{2}$ and all other chemicals were purchased from commercial sources and used as received unless otherwise specified. Horse blood serum was purchased from Sigma-Aldrich Korea (product No. H1138) and used as received. $3 \AA$ molecular sieves were activated at $240{ }^{\circ} \mathrm{C}$ under dynamic vacuum for overnight prior to use. Benzene, toluene, pentane, diethyl ether, and THF (tetrahydrofuran) was distilled from deep purple sodium benzophenone ketyl and stored over activated $3 \AA$ molecular sieves. Acetonitrile- $d_{3}\left(\mathrm{CD}_{3} \mathrm{CN}\right)$ was dried using activated $3 \AA$ molecular sieves. 


\section{Experimental Details}

Synthesis of $\left[2 \mathrm{a}^{\cdot+}\right]\left[\mathrm{BF}_{4}{ }^{-}\right]$

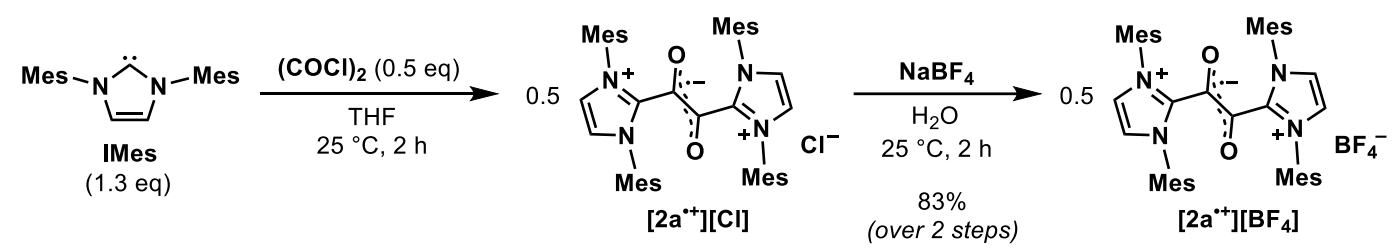

In a $\mathrm{N}_{2}$ atmosphere glovebox, IMes (200 $\mathrm{mg}, 0.66 \mathrm{mmol}, 1.3 \mathrm{eq}$ ) was placed in a $20 \mathrm{~mL}$ vial and subsequently dry THF $(4 \mathrm{~mL})$ was added to the vial. The solution was stirred at $25{ }^{\circ} \mathrm{C}$, and dry THF solution $(1 \mathrm{~mL})$ of oxalyl chloride $\left((\mathrm{COCl})_{2}, 21.6 \mu \mathrm{L}, 0.25 \mathrm{mmol}, 0.5 \mathrm{eq}\right)$ was slowly added. After 2 hours, the resulting precipitate was collected on a frit, washed with dry THF $(3 \times 3 \mathrm{~mL})$ and dry diethyl ether $(3 \times 3 \mathrm{~mL})$, and dried in vacuo to afford $\left[\mathbf{2 a}^{\mathbf{a}^{+}}\right]\left[\mathrm{Cl}^{-}\right]$as a purple solid. The obtained $\left[2 \mathbf{a}^{*+}\right]\left[\mathrm{Cl}^{-}\right]$was then suspended in distilled water $(5 \mathrm{~mL})$ and aqueous solution $(1 \mathrm{~mL})$ of sodium tetrafluoroborate $\left(\mathrm{NaBF}_{4}, 40 \mathrm{mg}\right)$ was added. After 2 hours, the purple precipitate was collected on a frit, washed with distilled water $(3 \times 3 \mathrm{~mL})$, and dried in vacuo to afford $158 \mathrm{mg}$ of the product ( $83 \%$ over 2 steps). Single crystals of $\left[\mathbf{2 a}^{\mathbf{a}}\right]\left[\mathbf{C l}^{-}\right]$suitable for X-ray crystallography were obtained from slow evaporation of water/acetone mixture solution. Single crystals of $\left[\mathbf{2 a}^{\mathbf{a}^{+}}\right]\left[\mathbf{B F}_{4}{ }^{-}\right]$suitable for X-ray crystallography were obtained from slow diffusion of pentane into dichloromethane solution. The radicals were NMR silent and their ${ }^{1} \mathrm{H}$ and ${ }^{13} \mathrm{C}$ spectra showed no meaningful data, except for a very broad peak in the $1 \mathrm{H}$ NMR around $2.5 \mathrm{ppm}$ which we assigned as $\mathrm{Ar}-\mathrm{CH}_{3}$ from mesityl substituents. Data for $\left[\mathbf{2 a}^{\cdot+}\right]\left[\mathbf{C l}^{-}\right]:{ }^{1} \mathrm{H}$ NMR $\left(500 \mathrm{MHz}\right.$, Acetonitrile- $\left.d_{3}\right) \delta 2.48$ (br s, Ar$\mathrm{CH}_{3}$ ) ppm. UV absorption $\lambda_{\max }=472 \mathrm{~nm}$ (water), $510 \mathrm{~nm}$ (acetonitrile), $521 \mathrm{~nm}$ (dichloromethane). EPR (microwave frequency $=9.4313 \mathrm{GHz}) g_{\text {iso }}=2.0062$; hyperfine coupling constants: $a\left({ }^{14} \mathrm{~N}\right)=$ 3.0, 3.0, 2.6, $2.6 \mathrm{MHz}$. Data for $\left[\mathbf{2 a}^{\cdot+}\right]\left[\mathbf{B F}_{4}^{-}\right]$: m.p. $305^{\circ} \mathrm{C}$ (decomp). IR(ATR): 1373 (C-O), 1052 (B-F) $\mathrm{cm}^{-1} .{ }^{19} \mathrm{~F}\left\{{ }^{1} \mathrm{H}\right\}$ NMR $\left(282 \mathrm{MHz}\right.$, Acetonitrile- $\left.d_{3}\right) \delta-151.9 \mathrm{ppm}$. Anal. Calcd for $\mathrm{C}_{44} \mathrm{H}_{48} \mathrm{BF}_{4} \mathrm{~N}_{4} \mathrm{O}_{2}: \mathrm{C}, 70.31 ; \mathrm{H}, 6.44 ; \mathrm{N}, 7.45$. Found: $\mathrm{C}, 69.99 ; \mathrm{H}, 7.01 ; \mathrm{N}, 7.48$. HRMS (FAB): m/z calcd for $\left[\mathrm{C}_{44} \mathrm{H}_{48} \mathrm{~N}_{4} \mathrm{O}_{2}(\mathrm{M})^{+}\right]$664.3777, found 664.3779. EPR (microwave frequency $=9.4467 \mathrm{GHz}$ ) $g_{\text {iso }}=2.0060$; hyperfine coupling constants: $a\left({ }^{14} \mathrm{~N}\right)=3.0,3.0,2.6,2.6 \mathrm{MHz}$.

\section{Synthesis of $\left[2 \mathrm{~b}^{\circ+}\right]\left[\mathrm{BF}_{4}^{-}\right]$}
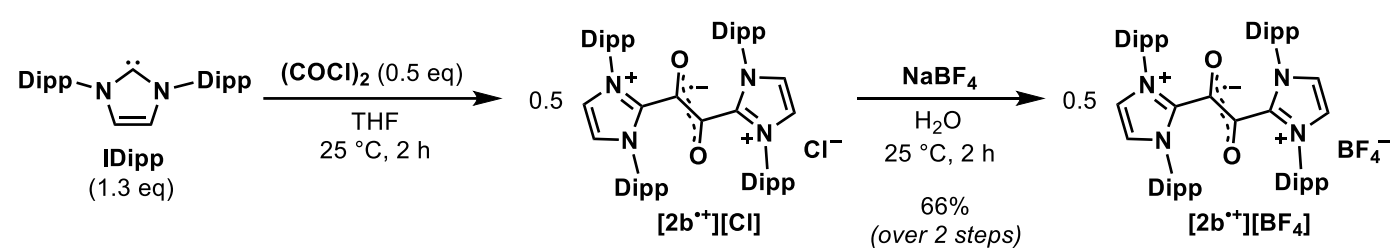

$\left[2 b^{*+}\right][\mathrm{Cl}]$

$66 \%$
(over 2 steps)

$\left[2 b^{\bullet+}\right]\left[B_{4}\right]$

In a $\mathrm{N}_{2}$ atmosphere glovebox, IDipp (100 mg, $0.26 \mathrm{mmol}, 1.3 \mathrm{eq}$ ) was placed in a $20 \mathrm{~mL}$ vial and subsequently dry THF $(2 \mathrm{~mL})$ was added to the vial. The solution was stirred at $25^{\circ} \mathrm{C}$, and dry THF solution $(1 \mathrm{~mL})$ of oxalyl chloride $\left((\mathrm{COCl})_{2}, 8.4 \mu \mathrm{L}, 0.10 \mathrm{mmol}, 0.5 \mathrm{eq}\right)$ was slowly added. After 2 hours, dry pentane $(15 \mathrm{~mL})$ was added, and the resulting precipitate was washed with diethyl ether $(2 \times 1 \mathrm{~mL})$ and dried in vacuo. Further washing with distilled water $(3 \times 3 \mathrm{~mL})$ afford $\left[2 \mathbf{b}^{\mathbf{}}\right]\left[\mathbf{C l}^{-}\right]$ as a purple solid. The obtained $\left[\mathbf{2 b}^{\circ+}\right]\left[\mathbf{C l}^{-}\right]$was then suspended in distilled water $(1 \mathrm{~mL})$ and aqueous 
solution $(1 \mathrm{~mL})$ of sodium tetrafluoroborate $\left(\mathrm{NaBF}_{4}, 15 \mathrm{mg}\right)$ was added. After 2 hours, the purple precipitate was collected on a frit, washed with distilled water $(3 \times 3 \mathrm{~mL})$, and dried in vacuo to afford $60.4 \mathrm{mg}$ of the product ( $66 \%$ over 2 steps). Single crystals of $\left[\mathbf{2} \mathbf{b}^{\mathbf{}}\right]\left[\mathbf{B F}_{4}^{-}\right]$suitable for X-ray crystallography were obtained from slow evaporation of water/acetone mixture solution. Data for $\left[2 \mathbf{b}^{\circ+}\right]\left[\mathbf{B F}_{4}{ }^{-}\right]$: m.p. $215^{\circ} \mathrm{C}$ (decomp). ${ }^{19} \mathrm{~F}\left\{{ }^{1} \mathrm{H}\right\}$ NMR $\left(282 \mathrm{MHz}\right.$, Acetonitrile- $\left.d_{3}\right) \delta-151.9$ ppm. Anal. Calcd for $\mathrm{C}_{56} \mathrm{H}_{72} \mathrm{BF}_{4} \mathrm{~N}_{4} \mathrm{O}_{2}: \mathrm{C}, 73.11 ; \mathrm{H}, 7.89 ; \mathrm{N}, 6.09$ (cf. $\mathrm{C}_{56} \mathrm{H}_{72} \mathrm{BF}_{4} \mathrm{~N}_{4} \mathrm{O}_{2} \cdot 2 \mathrm{H}_{2} \mathrm{O}: \mathrm{C}, 70.35 ; \mathrm{H}, 8.01$; $\mathrm{N}$, 5.86). Found: C, 70.39; H, 8.35; N, 5.67. HRMS (FAB): $\mathrm{m} / \mathrm{z}$ calcd for $\left[\mathrm{C}_{56} \mathrm{H}_{72} \mathrm{~N}_{4} \mathrm{O}_{2}(\mathrm{M})^{+}\right]$ 832.5655, found 832.5658. EPR (microwave frequency $=9.4413 \mathrm{GHz}$ ) $g_{\text {iso }}=2.0059$; hyperfine coupling constants: $a\left({ }^{14} \mathrm{~N}\right)=3.0,3.0,2.6,2.6 \mathrm{MHz}$.

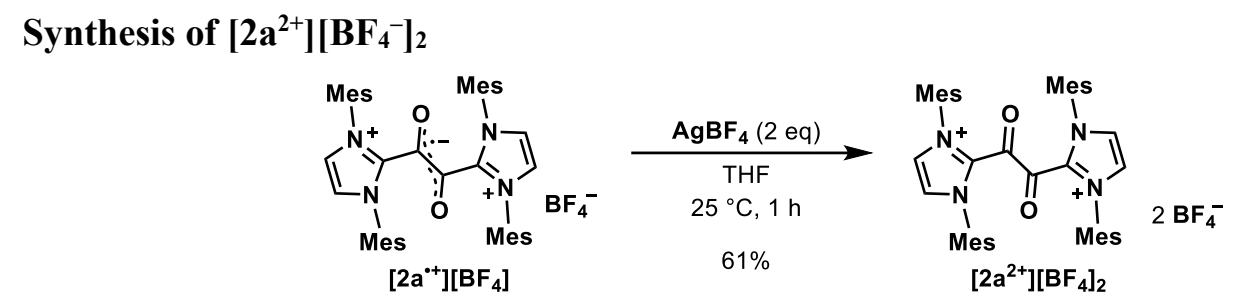

In a $\mathrm{N}_{2}$ atmosphere glovebox, $\left[\mathbf{2 a}^{\mathbf{a}^{+}}\right]\left[\mathbf{B F}_{4}{ }^{-}\right](30 \mathrm{mg}, 0.040 \mathrm{mmol})$ and silver(I) tetrafluoroborate $\left(\mathrm{AgBF}_{4}, 16 \mathrm{mg}, 0.080 \mathrm{mmol}, 2 \mathrm{eq}\right)$ were placed in a $4 \mathrm{~mL}$ vial and subsequently dry THF $(2 \mathrm{~mL})$ was added to the vial. After 1 hour, the reaction mixture was centrifuged and decanted, and the remaining brown precipitate was dissolved in dry dichloromethane $(3 \mathrm{~mL})$. The solution was filtered through Celite to remove silver containing byproducts, and the filtrate was concentrated in vacuo. Dry pentane $(3 \mathrm{~mL})$ was added and the resulting precipitate was washed with dry THF $(1 \times$ $1 \mathrm{~mL})$ and dry pentane $(2 \times 1 \mathrm{~mL})$, and dried in vacuo to afford $\left[\mathbf{2 a}^{2+}\right]\left[\mathbf{B F}_{4}{ }^{-}\right]_{2}$ as an orange solid. (20 mg, 61\%). ${ }^{1} \mathrm{H}$ NMR (500 MHz, Acetonitrile- $\left.d_{3}\right) \delta 7.97$ (s, 4H), $7.10(\mathrm{~s}, 8 \mathrm{H}), 2.44(\mathrm{~s}, 12 \mathrm{H}), 1.72$ (s, 24H) ppm. ${ }^{13} \mathrm{C}\left\{{ }^{1} \mathrm{H}\right\}$ NMR (126 MHz, Acetonitrile- $\left.d_{3}\right) \delta 172.4,144.1,135.6,135.4,131.3,130.5$, 129.6, 21.3, 17.2 ppm. ${ }^{19} \mathrm{~F}\left\{{ }^{1} \mathrm{H}\right\}$ NMR (470 MHz, Acetonitrile- $\left.d_{3}\right) \delta-151.8 \mathrm{ppm}$. Single crystals of $\left[\mathbf{2 a}^{2+}\right]\left[\mathbf{B F}_{4}^{-}\right]_{2}$ suitable for X-ray crystallography were obtained from dichloromethane/pentane solution. m.p. $270{ }^{\circ} \mathrm{C}$ (decomp). IR(ATR): 1699 (C-O), 1056 (B-F) $\mathrm{cm}^{-1}$. Anal. Calcd for $\mathrm{C}_{44} \mathrm{H}_{48} \mathrm{~B}_{2} \mathrm{~F}_{8} \mathrm{~N}_{4} \mathrm{O}_{2}$ : C, 63.03; H, $5.77\left(c f . \mathrm{C}_{44} \mathrm{H}_{48} \mathrm{~B}_{2} \mathrm{~F}_{8} \mathrm{~N}_{4} \mathrm{O}_{2} \cdot \mathrm{H}_{2} \mathrm{O}: \mathrm{C}, 61.70 ; \mathrm{H}, 5.88 ; \mathrm{N}, 6.54\right) ; \mathrm{N}, 6.68$. Found: C, 61.54; H, 6.24; N, 6.73 .

\section{Synthesis of $2 a^{0}$}
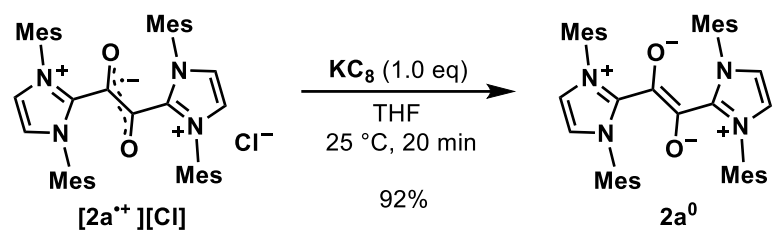

In a $\mathrm{N}_{2}$ atmosphere glovebox, $\left[\mathbf{2 a}^{\cdot+}\right]\left[\mathrm{Cl}^{-}\right](10 \mathrm{mg}, 0.014 \mathrm{mmol}, 1.0 \mathrm{eq})$ and potassium graphite $\left(\mathrm{KC}_{8}, 1.9 \mathrm{mg}, 0.014 \mathrm{mmol}, 1.0 \mathrm{eq}\right)$ were placed in a $4 \mathrm{~mL}$ vial and subsequently dry THF $(0.8 \mathrm{~mL})$ was added to the vial. The reaction mixture was quickly sonicated to dissolve the starting material. After 20 minutes at $25^{\circ} \mathrm{C}$, the reaction mixture was filtered to a thin pad of Celite and the resulting 
filtrate was dried in vacuo to afford $\mathbf{2 a}^{\mathbf{0}}$ as a dark green solid ( $\left.8.7 \mathrm{mg} ; 92 \%\right)$. Single crystals of $\mathbf{2} \mathbf{a}^{\mathbf{0}}$ suitable for X-ray crystallography were obtained from slow diffusion of pentane into the toluene solution. ${ }^{1} \mathrm{H}$ NMR $\left(850 \mathrm{MHz}\right.$, Benzene- $\left.d_{6}\right) \delta 6.77(\mathrm{~s}, 8 \mathrm{H}), 5.80(\mathrm{~s}, 4 \mathrm{H}), 2.26(\mathrm{~s}, 12 \mathrm{H}), 2.20(\mathrm{~s}, 24 \mathrm{H})$ ppm. ${ }^{13} \mathrm{C}\left\{{ }^{1} \mathrm{H}\right\}$ NMR $\left(214 \mathrm{MHz}\right.$, Benzene- $\left.d_{6}\right) \delta 137.0,126.6,125.4,124.8,129.2,128.8,119.1,21.3$, $18.6 \mathrm{ppm}$. UV absorption $\lambda_{\max }=394,581 \mathrm{~nm}$ (benzene).

\section{Mechanistic study}
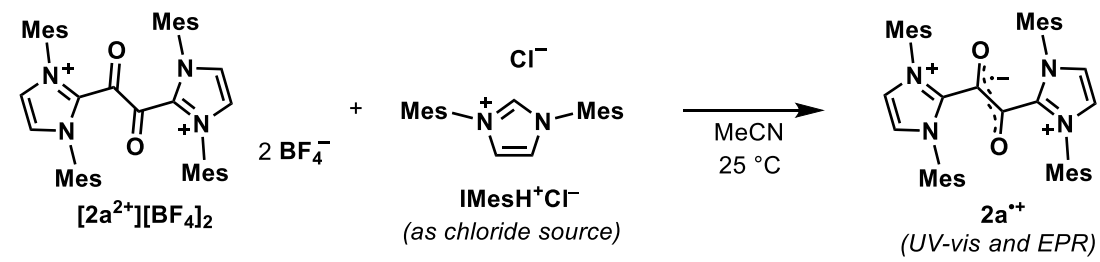

We proposed that the formation of $\mathbf{2 a}^{\mathbf{0}+}$ from IMes and oxalyl chloride involves the formation of $\mathbf{2 a}^{2+}$ as an intermediate followed by a spontaneous one electron reduction. Interestingly, the reaction does not require additional reducing agent, therefore chloride or NHC in the reaction mixture may act as electron source. (Single electron transfer from NHCs to electron acceptors have been recently reported by Severin and co-workers. $\left.{ }^{[2]}\right)$ Indeed, the reaction between $\left[\mathbf{2 a}^{2+}\right]\left[\mathbf{B F}_{4}{ }^{-}\right]_{2}$ with $\mathrm{IMesH}^{+} \mathrm{Cl}^{-}$ (as a soluble chloride source) afforded $\mathbf{2 \mathbf { a } ^ { + + }}$ which was detected by EPR and UV-vis spectroscopy.

In a $\mathrm{N}_{2}$ atmosphere glovebox, $\left[\mathbf{2 a}^{2+}\right]\left[\mathbf{B F}_{4}^{-}\right]_{2}(2.5 \mathrm{mg})$ and $\mathrm{IMesH}^{+} \mathrm{Cl}^{-}(5.0 \mathrm{mg}$, ca. 5 eq.) were dissolved in dry acetonitrile $(3 \mathrm{~mL})$. The reaction mixture was monitored by $\mathrm{UV}$-vis which showed the slow formation of $\mathbf{2} \mathbf{a}^{\mathbf{}+}$ (Figure S1). EPR study also showed the existence of $\mathbf{2} \mathbf{a}^{\mathbf{}}$ in the reaction mixture (Figure S2). The same reaction with tetrabutylammonium chloride as a chloride source instead of the imidazolium chloride gave identical results.

DFT calculation at M06/Def2-SV(P) level of theory with SMD model showed the electron transfer process from $\mathbf{2} \mathbf{a}^{2+}$ to chloride to generate $\mathbf{2} \mathbf{a}^{\cdot+}$ and $0.5 \mathrm{Cl}_{2}$ is thermodynamically favorable (6.9 kcal/mol in THF and $5.2 \mathrm{kcal} / \mathrm{mol}$ in acetonitrile). (Balanced equation: $\mathbf{2 a}^{2+} \cdot \mathrm{Cl}^{-} \rightarrow \mathbf{2 a}^{\bullet+}+0.5$ $\left.\mathrm{Cl}_{2}\right)$

(a)

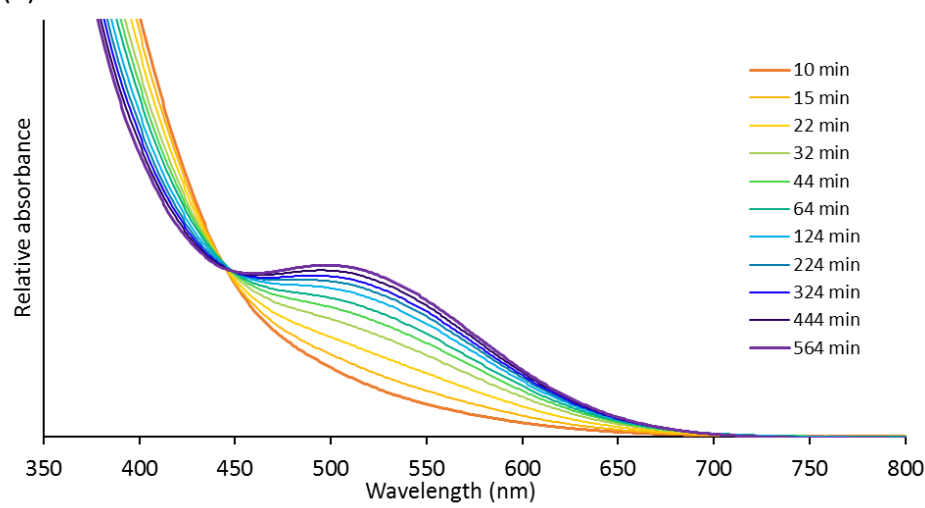

(b)

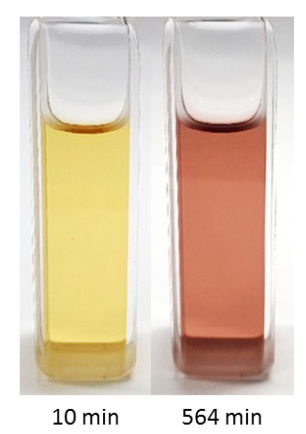

Figure S1. Monitoring of the formation of $\mathbf{2 a}^{++}$(corresponds to $\lambda_{\max }=510 \mathrm{~nm}$ in acetonitrile) from the reaction between $\left[\mathbf{2 a}^{2+}\right]\left[\mathbf{B F}_{4}^{-}\right]_{2}$ and $\mathrm{IMesH}^{+} \mathrm{Cl}^{-}$. (a) Selected spectra. (b) Color of the reaction mixture. 


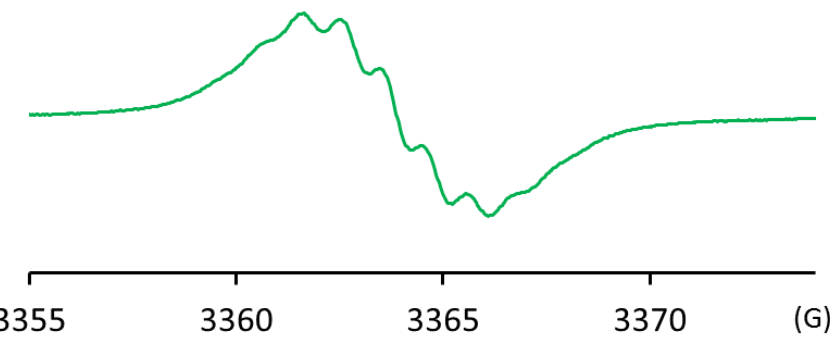

Figure S2. Experimental EPR spectra of the reaction mixture from the reaction between $\left[2 \mathbf{a}^{2+}\right]\left[\mathbf{B F}_{4}^{-}\right]_{2}$ and $\mathrm{IMesH}^{+} \mathrm{Cl}^{-}$at microwave frequency $=9.4452 \mathrm{GHz}$.

\section{Deuteration}

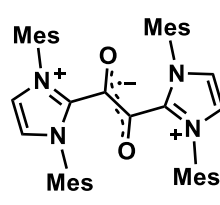

$2 a^{*+}$
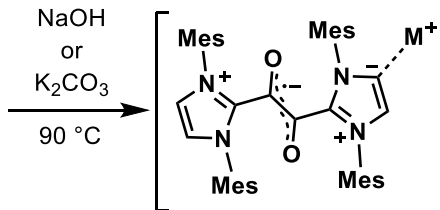

$(\mathbf{M}=\mathbf{N a}$ or $\mathbf{K})$

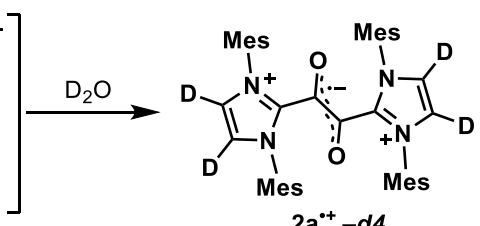

$2 a^{*+}-d 4$

To demonstrate the radical can undergo post-synthetic modification even under harsh condition, the imidazolium $\mathrm{C}-\mathrm{H}$ bonds of $\mathbf{2 a}^{\mathbf{*}}$ were deuterated. Under ambient atmosphere, $\left[\mathbf{2 a}^{\mathbf{*}}\right]\left[\mathbf{C l}^{-}\right](0.4$ $\mathrm{mg}$ ) and excess $\mathrm{NaOH}(11 \mathrm{mg})$ or $\mathrm{K}_{2} \mathrm{CO}_{3}(14 \mathrm{mg})$ were dissolved in $\mathrm{D}_{2} \mathrm{O}(1 \mathrm{~mL})$. The reaction mixture was heated to $90{ }^{\circ} \mathrm{C}$. No color change was observed during the reaction. After 2 hours, the reaction mixtures were diluted and analyzed by ESI-MS spectrometry (Figure S3 and Figure S4) which showed complete deuteration toward $\mathbf{2} \mathbf{a}^{\mathbf{}}-d_{4}$.
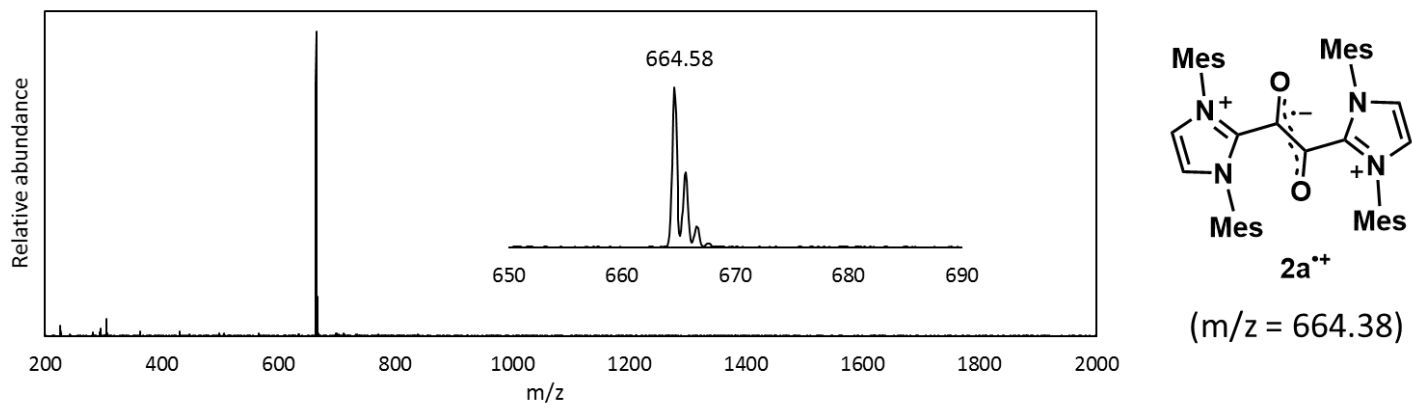

Figure S3. Experimental ESI-MS spectrum and expected mass of $\mathbf{2 a}^{\mathbf{}}$. 
(a)

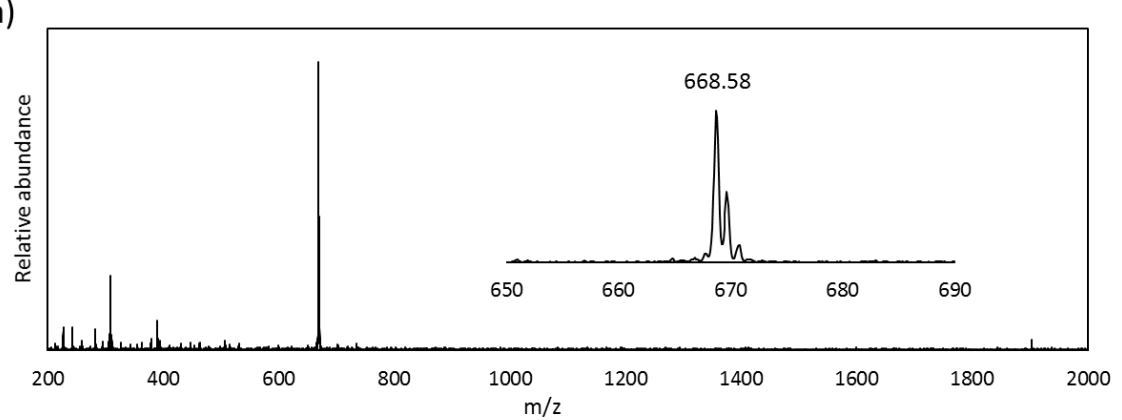

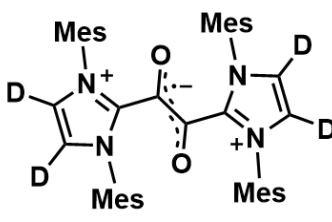

$2 a^{\bullet+}-d 4$

$(\mathrm{m} / \mathrm{z}=668.40)$

(b)

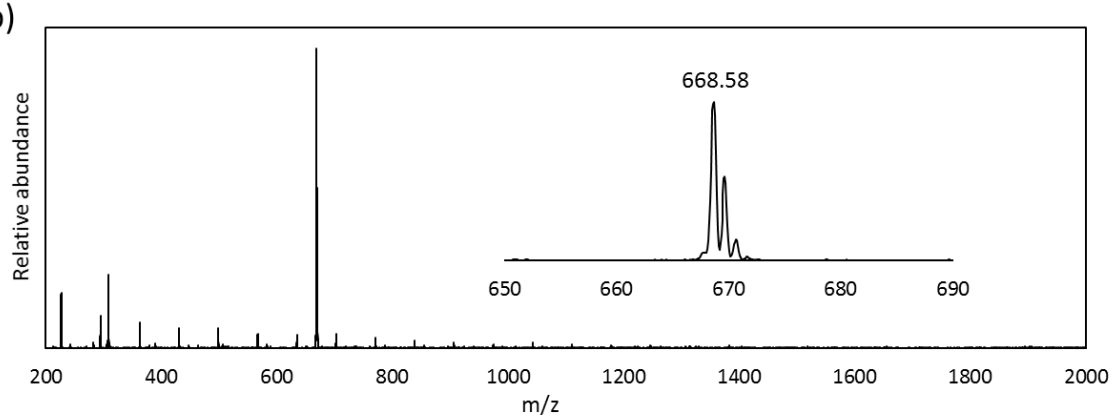

Figure S4. Experimental ESI-MS spectrum and expected mass of $\mathbf{2 a}^{\mathbf{* +}}-d_{4}$. (a) Deuteration with $\mathrm{K}_{2} \mathrm{CO}_{3}$. (b) Deuteration with $\mathrm{NaOH}$. 


\section{DFT Calculation}

\section{General information}

Geometry optimizations and frequency calculations were performed using Gaussian $16^{[3]}$ with M06 functional ${ }^{[4]}$ and either Def2-SV $(\mathrm{P})^{[5]}$ or 6-31G(d,p $)^{[6]}$ basis set. In the geometry optimization, the default tight convergence in the SCF cycle was used without any orbital symmetry constraints. Solvation free energies were calculated using the SMD model. ${ }^{[7]}$ Multiwfn $^{[8]}$ and IBOview ${ }^{[9]}$ programs were used to visualize Molecular orbitals. Wiberg bond indices (WBIs) were calculated from the optimized geometry with Löwdin orthogonalization method using Multiwfn.

\section{Coordinates of optimized structures}

The following optimized geometries were displayed in Cartesian coordinates (atomic unit). $\mathrm{E}^{\circ}$ represents the electronic energy of the optimized structure, and $\mathrm{G}^{\circ}$ represents the sum of electronic and thermal free energies in Hartree unit.

$$
\begin{aligned}
& \mathbf{2 a}^{\bullet+}\left[E^{\circ}=-2071.7768846 ; G^{\circ}=-2071.044877 ; G^{\circ}(T H F)=-2071.1192563 ;\right. \\
& \left.G^{\circ}(\mathrm{MeCN})=-2071.1267336\right] \operatorname{Def} 2-\mathrm{SV}(\mathrm{P})
\end{aligned}
$$

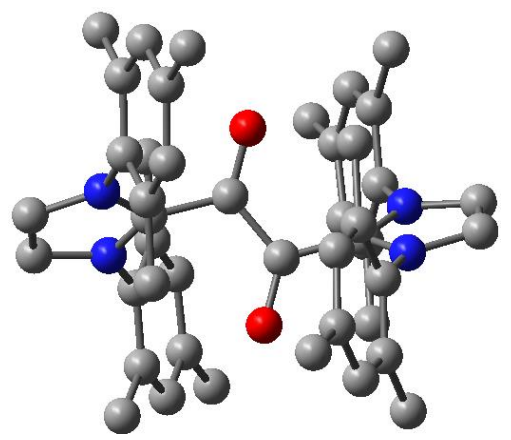

Charge $=1 ;$ Multiplicity $=2$;

\begin{tabular}{cccccc}
\hline & \multicolumn{2}{c}{ Atomic coordinates (Cartesian; in atomic unit) } & Mulliken charge & Spin density \\
\hline $\mathrm{O}$ & -0.0171840011 & 0.8480612757 & 1.5432186703 & -0.276975 & 0.322261 \\
$\mathrm{~N}$ & 0.9531572985 & 2.2329742473 & -1.4784521889 & -0.177772 & 0.009341 \\
$\mathrm{~N}$ & -0.9732981757 & 2.7149840828 & -0.6067925669 & -0.181867 & 0.010149 \\
$\mathrm{C}$ & 2.4091076556 & 0.9420679413 & -2.9519629225 & 0.086987 & 0.001577 \\
$\mathrm{C}$ & 2.2240605153 & 1.6070448015 & -1.7292426389 & -0.033928 & -0.006434 \\
$\mathrm{C}$ & 0.0674792475 & 0.6436473770 & 0.3141463414 & 0.057964 & 0.179057 \\
$\mathrm{C}$ & 0.0239353394 & 1.8160944269 & -0.6057497206 & 0.211481 & -0.013777 \\
$\mathrm{C}$ & 3.6594787054 & 0.3698623447 & -3.1785927654 & -0.267071 & 0.003125 \\
$\mathrm{C}$ & 4.4690840987 & 1.1616235332 & -1.0586920815 & -0.242914 & 0.003365 \\
$\mathrm{C}$ & 3.2287593904 & 1.7339466230 & -0.7630879147 & 0.085223 & 0.001990 \\
$\mathrm{C}$ & 4.7009967215 & 0.4707424115 & -2.2484056471 & 0.107455 & 0.000364 \\
$\mathrm{C}$ & 0.5381273926 & 3.4256784095 & -2.0427983886 & 0.012016 & 0.003733 \\
$\mathrm{C}$ & -2.2305941130 & 2.5600911385 & 0.0775994805 & -0.043861 & -0.008295 \\
$\mathrm{C}$ & -2.3963838824 & 3.1573589226 & 1.3315699438 & 0.105454 & 0.000265 \\
$\mathrm{C}$ & -3.2298121400 & 1.8136799597 & -0.5628971253 & 0.088162 & 0.003793 \\
$\mathrm{C}$ & 1.3143751224 & 0.8558351169 & -3.9700781496 & -0.278345 & 0.000132 \\
$\mathrm{C}$ & -0.6723327553 & 3.7241587113 & -1.5004434161 & 0.013144 & 0.000767 \\
$\mathrm{C}$ & 2.9946659818 & 2.4244000795 & 0.5467265236 & -0.294230 & -0.000158 \\
$\mathrm{C}$ & 6.0273876254 & -0.1670551985 & -2.5274792053 & -0.291789 & -0.000059 \\
$\mathrm{C}$ & -4.6760882124 & 2.2816219918 & 1.3497569549 & 0.122873 & -0.001051 \\
$\mathrm{C}$ & -4.4536717854 & 1.7015103860 & 0.0963008306 & -0.268962 & 0.002714 \\
$\mathrm{C}$ & -2.9850936263 & 1.1408374659 & -1.8800608933 & -0.298792 & 0.001719
\end{tabular}


Electronic Supplementary Information

\begin{tabular}{|c|c|}
\hline $\mathrm{C}$ & -3.6370766596 \\
\hline $\mathrm{C}$ & -1.2722244549 \\
\hline $\mathrm{C}$ & -6.0083484105 \\
\hline $\mathrm{O}$ & 0.0450386338 \\
\hline $\mathrm{N}$ & 0.9682139312 \\
\hline $\mathrm{N}$ & -0.9645257917 \\
\hline $\mathrm{C}$ & 2.4261572893 \\
\hline $\mathrm{C}$ & 2.2414972536 \\
\hline $\mathrm{C}$ & 0.0887147157 \\
\hline $\mathrm{C}$ & 0.0383976666 \\
\hline $\mathrm{C}$ & 3.6783486717 \\
\hline $\mathrm{C}$ & 4.4901718635 \\
\hline $\mathrm{C}$ & 3.2482470968 \\
\hline $\mathrm{C}$ & 4.7217101734 \\
\hline $\mathrm{C}$ & 0.5477861985 \\
\hline $\mathrm{C}$ & -2.2196681515 \\
\hline $\mathrm{C}$ & -2.3792667095 \\
\hline $\mathrm{C}$ & -3.2228628894 \\
\hline $\mathrm{C}$ & 1.3277353953 \\
\hline $\mathrm{C}$ & -0.6666785984 \\
\hline $\mathrm{C}$ & 3.0137801473 \\
\hline $\mathrm{C}$ & 6.0492842031 \\
\hline $\mathrm{C}$ & -4.6607342504 \\
\hline $\mathrm{C}$ & -4.4443003572 \\
\hline $\mathrm{C}$ & -2.9851946875 \\
\hline $\mathrm{C}$ & -3.6178478324 \\
\hline $\mathrm{C}$ & -1.2512251705 \\
\hline $\mathrm{C}$ & -5.9910309405 \\
\hline $\mathrm{H}$ & 3.8317012147 \\
\hline $\mathrm{H}$ & 5.2824065994 \\
\hline $\mathrm{H}$ & 1.1582939485 \\
\hline $\mathrm{H}$ & 0.3650257985 \\
\hline $\mathrm{H}$ & 1.5713978993 \\
\hline $\mathrm{H}$ & 1.1388005612 \\
\hline $\mathrm{H}$ & -1.3590170283 \\
\hline $\mathrm{H}$ & 2.3399054669 \\
\hline $\mathrm{H}$ & 3.9492546739 \\
\hline $\mathrm{H}$ & 2.5119923612 \\
\hline $\mathrm{H}$ & 5.9434354543 \\
\hline $\mathrm{H}$ & 6.7818766630 \\
\hline $\mathrm{H}$ & 6.4214581411 \\
\hline $\mathrm{H}$ & -5.2648045298 \\
\hline $\mathrm{H}$ & -2.5821577851 \\
\hline $\mathrm{H}$ & -3.9193244949 \\
\hline $\mathrm{H}$ & -2.2546236949 \\
\hline $\mathrm{H}$ & -3.7948824908 \\
\hline $\mathrm{H}$ & -0.4175994978 \\
\hline $\mathrm{H}$ & -1.5829656261 \\
\hline $\mathrm{H}$ & -0.9092277590 \\
\hline $\mathrm{H}$ & -6.7652338221 \\
\hline $\mathrm{H}$ & -5.9624728469 \\
\hline $\mathrm{H}$ & -6.3907559489 \\
\hline $\mathrm{H}$ & 3.8503194711 \\
\hline $\mathrm{H}$ & 5.3047999237 \\
\hline $\mathrm{H}$ & 1.1671362983 \\
\hline $\mathrm{H}$ & 0.3824224081 \\
\hline $\mathrm{H}$ & 1.5869475947 \\
\hline $\mathrm{H}$ & 1.1429291053 \\
\hline $\mathrm{H}$ & -1.3579454475 \\
\hline $\mathrm{H}$ & 2.3544107279 \\
\hline $\mathrm{H}$ & 3.9676266549 \\
\hline $\mathrm{H}$ & 2.5351253701 \\
\hline $\mathrm{H}$ & 5.9672830290 \\
\hline $\mathrm{H}$ & 6.8035153568 \\
\hline $\mathrm{H}$ & 6.4423589001 \\
\hline $\mathrm{H}$ & -5.2584021946 \\
\hline
\end{tabular}

2.9965040076

3.8971478463

2.1367095349

$-0.8532861446$

$-2.2346430762$

$-2.7055824915$

$-0.9418372919$

$-1.6128206725$

$-0.6426759186$

$-1.8137499237$

$-0.3737196800$

$-1.1813863376$

$-1.7502256497$

$-0.4842579349$

$-3.4233912138$

$-2.5501033664$

$-3.1459445462$

$-1.8067274646$

$-0.8438509937$

$-3.7151261959$

$-2.4463548319$

0.1516004232

$-2.2751666286$

$-1.6962746954$

$-1.1351507506$

$-2.9866973595$

$-3.8833086432$

$-2.1332838554$

$-0.1654768586$

1.2628719938

3.9516630015

0.5334715365

0.1288258259

1.8282621336

4.5547008771

3.3121660621

2.7583575514

1.7453003246

$-1.2716775135$

0.1028438816

0.1313432420

1.1437080324

1.8380279520

0.7101806190

0.3090113819

3.4466638132

3.2124856766

4.3122489858

4.7390517369

2.7934029938

2.4027326059

1.1014627539

0.1666981240

$-1.2892980079$

$-3.9512315958$

$-0.5163238818$

$-0.1161534696$

$-1.8132832466$

$-4.5410615212$

$-3.3303196345$

$-2.7873201826$

$-1.7687758814$

1.2564271204

$-0.1221056541$

$-0.1443211695$

$-1.1407521846$
1.9520802272

1.9866562079

2.0191393656

$-1.5451881478$

1.4780615542

0.6155195095

2.9474275757

1.7277742437

$-0.3160563645$

0.6085238373

3.1741596320

1.0609090934

0.7652188719

2.2470516488

2.0468923757

$-0.0728717905$

$-1.3285783608$

0.5650823993

3.9607146171

1.5100236738

$-0.5413489036$

2.5247718984

$-1.3540590849$

$-0.0989784656$

1.8840796445

$-1.9537064162$

$-1.9800346042$

$-2.0279831065$

$-4.1221151064$

$-0.3276848581$

$-2.7685913803$

$-3.5048046940$

$-4.7589619131$

$-4.4710468634$

$-1.6656395769$

0.4497993634

0.9899797818

1.2803583564

$-2.5420020339$

$-1.7673387793$

$-3.5166129787$

$-0.3913640467$

$-2.6409819950$

$-2.2807079016$

$-1.7900628757$

2.9408577139

2.1572794469

2.9600787092

1.3654504647

1.5483151435

3.0893594565

1.9378911605

4.1148385297

0.3322310723

2.7720554134

3.4903323542

4.7482510592

4.4641594120

1.6791221961

$-0.4411274991$

$-0.9807433443$

$-1.2790313149$

2.5355810917

1.7657572082

3.5150535168

0.3863361519
$-0.264024$

$-0.288616$

$-0.293721$

$-0.274910$

$-0.178061$

$-0.181705$

0.085212

$-0.035259$

0.060895

0.212034

$-0.266952$

$-0.244279$

0.086841

0.107347

0.012035

$-0.045460$

0.104887

0.088059

$-0.279997$

0.013084

$-0.294767$

$-0.291574$

0.122729

$-0.268678$

$-0.300387$

$-0.263966$

$-0.289807$

$-0.293847$

0.070696

0.055156

0.148413

0.155291

0.131561

0.134084

0.148673

0.123237

0.137414

0.178205

0.142645

0.128512

0.149157

0.056835

0.133316

0.132498

0.173171

0.070789

0.159792

0.131167

0.129980

0.153500

0.133675

0.133179

0.070891

0.056081

0.148491

0.157241

0.131517

0.134200

0.148758

0.123134

0.137352

0.178392

0.142702

0.128572

0.149333

0.056907
0.002089

0.000063

0.000045

0.317473

0.008064

0.008586

0.001568

$-0.005856$

0.174180

$-0.013737$

0.002665

0.002927

0.001617

0.000506

0.003265

$-0.007569$

0.000113

0.003239

0.000243

0.000432

0.000190

$-0.000064$

$-0.001094$

0.002873

0.001773

0.002218

0.000106

0.000045

$-0.000165$

$-0.000145$

$-0.000102$

$-0.001472$

0.000030

0.000267

0.000160

0.000351

0.000098

$-0.002899$

0.000036

$-0.000002$

0.000022

$-0.000148$

0.000209

0.000126

$-0.004960$

$-0.000099$

$-0.001657$

$-0.000003$

$-0.000009$

$-0.000033$

$-0.000004$

$-0.000018$

$-0.000144$

$-0.000118$

$-0.000066$

$-0.001677$

0.000024

0.000299

0.000180

0.000304

0.000074

$-0.002769$

0.000040

$-0.000001$

0.000026

$-0.000154$ 


$\begin{array}{lll}\mathrm{H} & -2.5832789483 & -1.8323578259 \\ \mathrm{H} & -3.9224275095 & -0.7079294588 \\ \mathrm{H} & -2.2561490394 & -0.3016188725 \\ \mathrm{H} & -3.7709799198 & -3.4359122512 \\ \mathrm{H} & -0.3967774988 & -3.1974242456 \\ \mathrm{H} & -1.5579122551 & -4.2985835004 \\ \mathrm{H} & -0.8893112421 & -4.7249477061 \\ \mathrm{H} & -6.7460036247 & -2.7973811929 \\ \mathrm{H} & -5.9393347552 & -2.3923445976 \\ \mathrm{H} & -6.3798044011 & -1.1008649674\end{array}$

2.6455216867
2.2814253008
1.7982068532
-2.9436478978
-2.1474080548
-2.9546713922
-1.3577772067
-1.5644968419
-3.0996591041
-1.9416280357

0.132888

0.131950

0.175440

0.070847

0.161032

0.131215

0.130087

0.153709

0.133706

0.133241
0.000181

0.000103

$-0.004527$

$-0.000109$

$-0.001722$

$-0.000004$

$-0.000003$

$-0.000034$

$-0.000004$

$-0.000018$

$\mathbf{2 a}^{\cdot+}\left[E^{\circ}=-2073.4773383 ; G^{\circ}=-2072.741530\right] 6-31 G(d, p)$

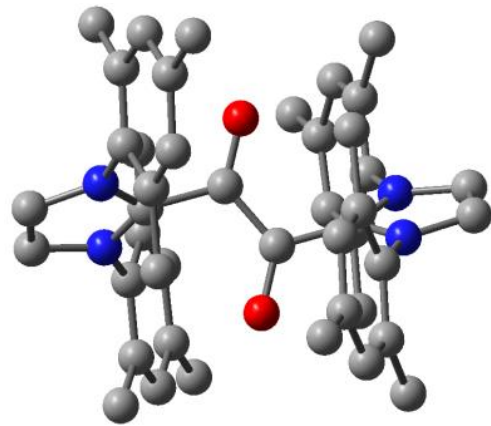

Charge $=1 ;$ Multiplicity $=2$;

\begin{tabular}{|c|c|c|c|c|}
\hline & \multicolumn{3}{|c|}{ Atomic coordinates (Cartesian; in atomic unit) } & $\begin{array}{l}\text { Isotropic Fermi Contact Couplings } \\
\text { (Hyperfine coupling constants; } \mathrm{MHz} \text { ) }\end{array}$ \\
\hline $\mathrm{O}$ & 0.040199 & 0.865488 & 1.539833 & -39.1021 \\
\hline $\mathrm{N}$ & 0.96107 & 2.219707 & -1.49551 & 3.03327 \\
\hline $\mathrm{N}$ & -0.96978 & 2.694625 & -0.63654 & 2.80036 \\
\hline $\mathrm{C}$ & 2.429092 & 0.928759 & -2.95187 & 0.12768 \\
\hline $\mathrm{C}$ & 2.239687 & 1.600492 & -1.73708 & -1.06926 \\
\hline $\mathrm{C}$ & 0.083386 & 0.640677 & 0.307733 & -6.72775 \\
\hline $\mathrm{C}$ & 0.032062 & 1.802708 & -0.624 & -12.5269 \\
\hline $\mathrm{C}$ & 3.684571 & 0.37504 & -3.17761 & 0.24807 \\
\hline $\mathrm{C}$ & 4.482676 & 1.187367 & -1.06547 & 0.10855 \\
\hline $\mathrm{C}$ & 3.237514 & 1.742408 & -0.77134 & -0.38423 \\
\hline $\mathrm{C}$ & 4.723398 & 0.500193 & -2.25158 & -0.01659 \\
\hline $\mathrm{C}$ & 0.539097 & 3.405743 & -2.07186 & -0.20797 \\
\hline $\mathrm{C}$ & -2.22349 & 2.545896 & 0.06017 & -0.30265 \\
\hline $\mathrm{C}$ & -2.37119 & 3.154686 & 1.307593 & 0.0212 \\
\hline $\mathrm{C}$ & -3.22576 & 1.793501 & -0.56027 & -0.32943 \\
\hline $\mathrm{C}$ & 1.327708 & 0.806745 & -3.95927 & -0.32037 \\
\hline $\mathrm{C}$ & -0.67227 & 3.698887 & -1.53876 & -0.36754 \\
\hline $\mathrm{C}$ & 2.995511 & 2.426995 & 0.53979 & -0.29068 \\
\hline $\mathrm{C}$ & 6.066744 & -0.09864 & -2.53726 & -0.0225 \\
\hline $\mathrm{C}$ & -4.64795 & 2.291911 & 1.355623 & -0.10355 \\
\hline $\mathrm{C}$ & -4.44058 & 1.693861 & 0.111783 & 0.23045 \\
\hline $\mathrm{C}$ & -2.99657 & 1.0966 & -1.8676 & 0.40566 \\
\hline $\mathrm{C}$ & -3.6035 & 3.00742 & 1.940298 & 0.21428 \\
\hline $\mathrm{C}$ & -1.23486 & 3.889045 & 1.947738 & -0.38965 \\
\hline $\mathrm{C}$ & -5.97477 & 2.165789 & 2.039837 & 0.01886 \\
\hline $\mathrm{O}$ & -0.02901 & -0.86583 & -1.54115 & -39.6671 \\
\hline $\mathrm{N}$ & 0.943923 & -2.22109 & 1.493849 & 3.06855 \\
\hline $\mathrm{N}$ & -0.98048 & -2.70828 & 0.625841 & 2.81906 \\
\hline $\mathrm{C}$ & 2.406547 & -0.93065 & 2.956443 & 0.05948 \\
\hline $\mathrm{C}$ & 2.21881 & -1.59542 & 1.737685 & -1.12132 \\
\hline $\mathrm{C}$ & 0.059849 & -0.64664 & -0.30947 & -6.12269 \\
\hline $\mathrm{C}$ & 0.015712 & -1.8093 & 0.618411 & -12.7255 \\
\hline $\mathrm{C}$ & 3.658768 & -0.3701 & 3.183205 & 0.30851 \\
\hline $\mathrm{C}$ & 4.457391 & -1.16135 & 1.063536 & 0.15268 \\
\hline $\mathrm{C}$ & 3.21542 & -1.72322 & 0.768676 & -0.41422 \\
\hline $\mathrm{C}$ & 4.696393 & -0.48152 & 2.254162 & -0.03615 \\
\hline
\end{tabular}


Electronic Supplementary Information

\begin{tabular}{|c|c|c|c|c|}
\hline $\mathrm{C}$ & 0.527256 & -3.41054 & 2.066906 & -0.20771 \\
\hline $\mathrm{C}$ & -2.23707 & -2.55894 & -0.06542 & -0.34175 \\
\hline $\mathrm{C}$ & -2.39346 & -3.16979 & -1.31051 & 0.05087 \\
\hline $\mathrm{C}$ & -3.23288 & -1.8002 & 0.557471 & -0.27335 \\
\hline $\mathrm{C}$ & 1.308518 & -0.82459 & 3.969005 & -0.35005 \\
\hline $\mathrm{C}$ & -0.68012 & -3.71097 & 1.528627 & -0.38311 \\
\hline $\mathrm{C}$ & 2.976891 & -2.40289 & -0.54586 & -0.43237 \\
\hline $\mathrm{C}$ & 6.037244 & 0.121443 & 2.542952 & -0.01957 \\
\hline $\mathrm{C}$ & -4.66619 & -2.2959 & -1.35056 & -0.10034 \\
\hline $\mathrm{C}$ & -4.45032 & -1.69598 & -0.10912 & 0.22887 \\
\hline $\mathrm{C}$ & -2.99397 & -1.1017 & 1.862334 & 0.40721 \\
\hline $\mathrm{C}$ & -3.62796 & -3.01833 & -1.93783 & 0.21007 \\
\hline $\mathrm{C}$ & -1.26308 & -3.90952 & -1.95464 & -0.40172 \\
\hline $\mathrm{C}$ & -5.99501 & -2.16418 & -2.02978 & 0.0184 \\
\hline $\mathrm{H}$ & 3.861292 & -0.16347 & -4.10876 & -0.16393 \\
\hline $\mathrm{H}$ & 5.287225 & 1.297241 & -0.33796 & -0.09448 \\
\hline $\mathrm{H}$ & 1.152696 & 3.9249 & -2.79351 & 0.05909 \\
\hline $\mathrm{H}$ & 0.399388 & 0.473963 & -3.48075 & 0.62076 \\
\hline $\mathrm{H}$ & 1.593544 & 0.080535 & -4.73212 & 0.05441 \\
\hline $\mathrm{H}$ & 1.128899 & 1.760409 & -4.4649 & 0.41554 \\
\hline $\mathrm{H}$ & -1.3581 & 4.515526 & -1.71201 & 0.45181 \\
\hline $\mathrm{H}$ & 2.325821 & 3.291296 & 0.446833 & 0.08303 \\
\hline $\mathrm{H}$ & 3.93826 & 2.778351 & 0.969791 & 0.1442 \\
\hline $\mathrm{H}$ & 2.536728 & 1.739776 & 1.266414 & 1.03193 \\
\hline $\mathrm{H}$ & 5.985591 & -1.17161 & -2.74985 & 0.08202 \\
\hline $\mathrm{H}$ & 6.754468 & 0.029197 & -1.69469 & -0.00402 \\
\hline $\mathrm{H}$ & 6.529565 & 0.363411 & -3.417 & 0.10322 \\
\hline $\mathrm{H}$ & -5.2512 & 1.132246 & -0.35366 & -0.13496 \\
\hline $\mathrm{H}$ & -2.58444 & 1.769409 & -2.63065 & 0.0075 \\
\hline $\mathrm{H}$ & -3.93324 & 0.683325 & -2.25378 & 0.23069 \\
\hline $\mathrm{H}$ & -2.28814 & 0.260386 & -1.76461 & 0.95065 \\
\hline $\mathrm{H}$ & -3.74952 & 3.46485 & 2.918302 & -0.13807 \\
\hline $\mathrm{H}$ & -0.40014 & 3.197263 & 2.12691 & 0.31872 \\
\hline $\mathrm{H}$ & -1.53836 & 4.322299 & 2.904287 & 0.0125 \\
\hline $\mathrm{H}$ & -0.86246 & 4.704465 & 1.314878 & 0.0345 \\
\hline $\mathrm{H}$ & -6.70684 & 2.8608 & 1.610873 & -0.08795 \\
\hline $\mathrm{H}$ & -5.90193 & 2.385563 & 3.109134 & -0.01166 \\
\hline $\mathrm{H}$ & -6.38871 & 1.156794 & 1.926018 & -0.04076 \\
\hline $\mathrm{H}$ & 3.834166 & 0.162273 & 4.118118 & -0.20221 \\
\hline $\mathrm{H}$ & 5.260915 & -1.26062 & 0.333379 & -0.13193 \\
\hline $\mathrm{H}$ & 1.141523 & -3.92722 & 2.789741 & -0.02501 \\
\hline $\mathrm{H}$ & 0.376301 & -0.49551 & 3.496193 & 0.56775 \\
\hline $\mathrm{H}$ & 1.57159 & -0.10146 & 4.745667 & 0.06154 \\
\hline $\mathrm{H}$ & 1.118993 & -1.78311 & 4.468931 & 0.3358 \\
\hline $\mathrm{H}$ & -1.36143 & -4.53201 & 1.698952 & 0.39947 \\
\hline $\mathrm{H}$ & 2.311915 & -3.27121 & -0.45705 & 0.13646 \\
\hline $\mathrm{H}$ & 3.921587 & -2.74734 & -0.97731 & 0.20433 \\
\hline $\mathrm{H}$ & 2.515591 & -1.71499 & -1.27017 & 1.15442 \\
\hline $\mathrm{H}$ & 5.951868 & 1.192428 & 2.763622 & 0.07173 \\
\hline $\mathrm{H}$ & 6.725073 & 0.002376 & 1.699173 & -0.00505 \\
\hline $\mathrm{H}$ & 6.502641 & -0.3451 & 3.41897 & 0.09285 \\
\hline $\mathrm{H}$ & -5.256 & -1.12916 & 0.358574 & -0.11866 \\
\hline $\mathrm{H}$ & -2.58551 & -1.77614 & 2.625942 & 0.0387 \\
\hline $\mathrm{H}$ & -3.92584 & -0.67953 & 2.250436 & 0.2866 \\
\hline $\mathrm{H}$ & -2.27948 & -0.27109 & 1.75531 & 1.13823 \\
\hline $\mathrm{H}$ & -3.78045 & -3.47699 & -2.91428 & -0.12524 \\
\hline $\mathrm{H}$ & -0.42581 & -3.22147 & -2.13554 & 0.30622 \\
\hline $\mathrm{H}$ & -1.57145 & -4.34006 & -2.91086 & 0.01721 \\
\hline $\mathrm{H}$ & -0.89287 & -4.72754 & -1.32393 & 0.02485 \\
\hline $\mathrm{H}$ & -6.72762 & -2.8584 & -1.60042 & -0.08622 \\
\hline $\mathrm{H}$ & -5.92637 & -2.38118 & -3.09993 & -0.01136 \\
\hline $\mathrm{H}$ & -6.40561 & -1.1543 & -1.91181 & -0.04056 \\
\hline
\end{tabular}


$\mathbf{2 a}^{2+}\left[E^{\circ}=-2071.4621977 ; G^{\circ}=-2070.733418\right] \operatorname{Def} 2-S V(P)$

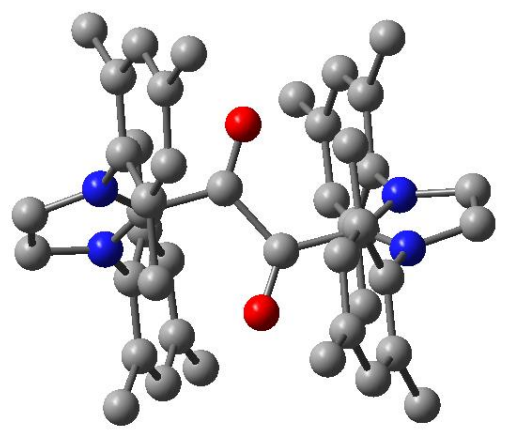

Charge $=2 ;$ Multiplicity $=1$;

\begin{tabular}{|c|c|c|c|}
\hline \multicolumn{4}{|c|}{ Atomic coordinates (Cartesian; in atomic unit) } \\
\hline $\mathrm{O}$ & -0.1905393215 & 0.7807802425 & 1.4919768034 \\
\hline $\mathrm{N}$ & 0.9120797792 & 2.2614998348 & -1.480069668 \\
\hline $\mathrm{N}$ & -1.0021782435 & 2.7656224305 & -0.5660578252 \\
\hline $\mathrm{C}$ & 2.3696970861 & 0.9921641829 & -2.9749774886 \\
\hline $\mathrm{C}$ & 2.1818919444 & 1.6232572344 & -1.7327683588 \\
\hline $\mathrm{C}$ & 0.0341725764 & 0.6803383683 & 0.3145461917 \\
\hline $\mathrm{C}$ & -0.0055729663 & 1.8656302091 & -0.588111266 \\
\hline $\mathrm{C}$ & 3.617705931 & 0.4171417241 & -3.2034364553 \\
\hline $\mathrm{C}$ & 4.4231277602 & 1.1645734566 & -1.0634889168 \\
\hline $\mathrm{C}$ & 3.1853084843 & 1.7375931435 & -0.7599728726 \\
\hline $\mathrm{C}$ & 4.6581545826 & 0.4965140755 & -2.2664915457 \\
\hline $\mathrm{C}$ & 0.4967764107 & 3.4488348839 & -2.0335305503 \\
\hline $\mathrm{C}$ & -2.2680060412 & 2.611016062 & 0.1179985062 \\
\hline $\mathrm{C}$ & -2.4569018524 & 3.2662171024 & 1.3420600272 \\
\hline $\mathrm{C}$ & -3.2486108548 & 1.8265403976 & -0.5065625783 \\
\hline $\mathrm{C}$ & 1.2993956902 & 0.9607124522 & -4.0229600819 \\
\hline $\mathrm{C}$ & -0.711846264 & 3.7553211504 & -1.4715922955 \\
\hline $\mathrm{C}$ & 2.9708053602 & 2.4375601104 & 0.5499493392 \\
\hline $\mathrm{C}$ & 5.9887796129 & -0.118579606 & -2.5650706671 \\
\hline $\mathrm{C}$ & -4.7186463735 & 2.3374603259 & 1.3799794883 \\
\hline $\mathrm{C}$ & -4.4746951925 & 1.7159902535 & 0.1506671472 \\
\hline $\mathrm{C}$ & -3.0000546394 & 1.0989043486 & -1.7954031644 \\
\hline $\mathrm{C}$ & -3.6967847336 & 3.0997196251 & 1.9591253493 \\
\hline $\mathrm{C}$ & -1.3744916151 & 4.0981141047 & 1.9575642164 \\
\hline $\mathrm{C}$ & -6.0517900204 & 2.2069273578 & 2.0460163471 \\
\hline $\mathrm{O}$ & 0.1089312485 & -0.8331864975 & -1.5302097138 \\
\hline $\mathrm{N}$ & 1.0170639204 & -2.2642671167 & 7156374 \\
\hline $\mathrm{N}$ & -0.9054292239 & -2.76 & 111558 \\
\hline $\mathrm{C}$ & 2.4491673635 & -0.949 & 443589 \\
\hline $\mathrm{C}$ & 2.2818954777 & 2168 & 2148096 \\
\hline $\mathrm{C}$ & 0.150101779 & 0629 & 8190213 \\
\hline $\mathrm{C}$ & 0.0964265439 & -1.8747341842 & 0.5878662317 \\
\hline $\mathrm{C}$ & 3.6912650618 & -0.3645041683 & 3.1884132268 \\
\hline $\mathrm{C}$ & 4.5321601542 & -1.164746048 & 1.0813661214 \\
\hline $\mathrm{C}$ & 3.3017185358 & -1.7505612928 & 0.7732437475 \\
\hline $\mathrm{C}$ & 4.7466166812 & -0.4654584004 & 2.2704223253 \\
\hline $\mathrm{C}$ & 0.5978039607 & -3.4453438174 & 2.0465731705 \\
\hline $\mathrm{C}$ & -2.1664023936 & -2.6118756605 & -0.111297417 \\
\hline $\mathrm{C}$ & -2.3294245736 & -3.2451926897 & -1.351483905 \\
\hline $\mathrm{C}$ & -3.1775218445 & -1.875481881 & 0.5259932293 \\
\hline $\mathrm{C}$ & 1.3596595661 & -0.8936018763 & 3.9823514038 \\
\hline $\mathrm{C}$ & -0.6138246963 & -3.7510035117 & 1.4956031062 \\
\hline $\mathrm{C}$ & 3.1116601687 & -2.4750150097 & -0.5259109181 \\
\hline & 6.0731703516 & 0.1550037705 & 2.5757059491 \\
\hline$\tau$ & -4.6153078403 & -2.3789246373 & -1.3887741769 \\
\hline$C$ & -4.4007072622 & -1.786215769 & -0.1400828632 \\
\hline & -2.9792602531 & -1.1834784513 & 1.8423827416 \\
\hline & -3.5682112462 & -3.0982879139 & -1.9765180602 \\
\hline
\end{tabular}

C $\quad-1.2255341934$

C -5.9353519944

H 3.795304018

H 5.2377824306

H 1.1087437445

H $\quad 0.2934175732$

H 1.4910463578

H 1.2680299017

H $\quad-1.4014683607$

H $\quad 2.2787163279$

H 3.9271593845

H 2.5705125485

H 5.8948363457

H 6.7072278195

H $\quad 6.4351269684$

H -5.2728811938

H -2.321545076

H -3.9470815226

H $\quad-2.562568527$

H $\quad-3.8748613503$

H -0.4423356438

H $\quad-1.6812894189$

H -1.1249112723

H -6.7603135712

H $\quad-5.9833481663$

H $\quad-6.5068558457$

H 3.8518413516

H 5.3569520646

H 1.2109080757

H $\quad 0.362796275$

H 1.5471452714

H 1.3063287607

H -1.3062848188

H 2.3708499154

H 4.063431273

H 2.7853670829

H 5.965192326

H 6.7668631486

H 6.559727333

H $\quad-5.2242220807$

H $\quad-2.2200028706$

H $\quad-3.9251997998$

H $\quad-2.6708363829$

H -3.7271801915

H -0.313088532

H -1.5263514806

H $\quad-0.9463358607$

H $\quad-6.775766233$

H -6.0477950813

H -6.051947409
$-4.0453916547$

$-2.2295393728$

$-0.0948135551$

1.2610670765

3.9762087597

0.7952331973

0.158123986

1.9084021574

4.5833720159

3.2982036719

2.8243849583

1.7520872507

$-1.2053634289$

0.0272778782

0.3184518152

1.1285711101

1.6411950935

0.9281702913

0.0925644488

3.5907227908

3.5165235333

4.4776699335

4.9792693068

2.9636257063

2.3597669412

1.2172613798

0.1738810949

$-1.2718303378$

$-3.9658633107$

$-0.7231358841$

$-0.0855078227$

$-1.8346439314$

$-4.5746203335$

$-3.2948792216$

$-2.922013509$

$-1.7866731273$

1.2185632762

0.0929569897

$-0.3498306988$

$-1.2464010278$

$-1.6654714763$

$-1.1544149255$

$-0.1261599197$

$-3.5796658222$

$-3.4354692238$

$-4.4358371801$

$-4.9169203196$

$-2.3876930565$

$-2.9387657397$

$-1.2088039895$
$-1.9712798036$

$-2.077383503$

$-4.1585638751$

$-0.3340385313$

$-2.7666175507$

$-3.5964951615$

$-4.7547661652$

$-4.5960044332$

$-1.6434178505$

0.4728686729

0.9429350705

1.3266343693

$-2.7533502056$

$-1.7398068817$

$-3.4778617178$

$-0.3231177166$

$-2.4820357818$

$-2.3367142479$

$-1.6201584217$

2.9243945358

2.0974608455

2.9459809297

1.3348442058

1.6566934807

3.1367556681

1.8592909712

4.1319491494

0.3642857759

2.7835492802

3.5357274003

4.7091306478

4.5646469517

1.6766343942

$-0.4642658655$

$-0.8623822922$

$-1.3340531162$

2.8605454254

1.7191303306

3.4318388742

0.3476185428

2.4871289953

2.4118497102

1.7016172366

$-2.9497900516$

$-2.1173030739$

$-2.9572708869$

$-1.3476392023$

$-1.3777875236$

$-2.9146013614$

$-2.4929074661$ 


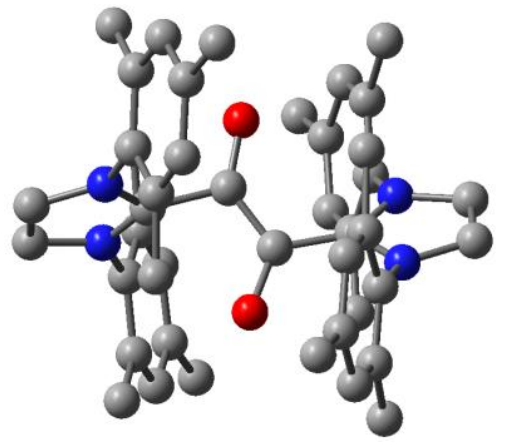

Charge $=0$; Multiplicity $=1$;

\begin{tabular}{|c|c|c|c|}
\hline \multicolumn{4}{|c|}{ Atomic coordinates (Cartesian; in atomic unit) } \\
\hline $\mathrm{O}$ & -0.3565716492 & 0.6753540214 & 1.5088442149 \\
\hline $\mathrm{N}$ & 0.7784339796 & 2.0556572567 & -1.6156509422 \\
\hline $\mathrm{N}$ & -0.9674782033 & 2.7239449448 & -0.4720818403 \\
\hline $\mathrm{C}$ & 2.3173636567 & 0.8067365072 & -3.042092108 \\
\hline $\mathrm{C}$ & 2.0660186046 & 1.4750089349 & -1.8358291168 \\
\hline $\mathrm{C}$ & -0.0290519391 & 0.5534449037 & 0.2513507283 \\
\hline $\mathrm{C}$ & -0.0672165233 & 1.702971981 & -0.6037858379 \\
\hline $\mathrm{C}$ & 3.5980563428 & 0.2886231565 & -3.2399066782 \\
\hline $\mathrm{C}$ & 4.3094054117 & 1.0987808025 & -1.0945217495 \\
\hline $\mathrm{C}$ & 3.0429922362 & 1.6326274774 & -0.8431719918 \\
\hline $\mathrm{C}$ & 4.602834658 & 0.4174615871 & -2.2774799527 \\
\hline $\mathrm{C}$ & 0.388550892 & 3.2905173992 & -2.1258781507 \\
\hline $\mathrm{C}$ & -2.2308545236 & 2.5810847928 & 0.1869854236 \\
\hline $\mathrm{C}$ & -2.4011427426 & 3.1021742002 & 1.4729521588 \\
\hline $\mathrm{C}$ & -3.2540457348 & 1.9117958489 & -0.4989278674 \\
\hline $\mathrm{C}$ & 1.2340536952 & 0.6319838923 & -4.0579679945 \\
\hline $\mathrm{C}$ & -0.6937830387 & 3.6986537297 & -1.4221654438 \\
\hline $\mathrm{C}$ & 2.7510952995 & 2.3460021118 & 0.4435497533 \\
\hline $\mathrm{C}$ & 5.9549157273 & -0.1916102395 & -2.5004420277 \\
\hline $\mathrm{C}$ & -4.7201148701 & 2.3436618746 & 1.4042661978 \\
\hline $\mathrm{C}$ & -4.4990682551 & 1.822394384 & 0.1265405906 \\
\hline $\mathrm{C}$ & -3.005598372 & 1.2744765507 & -1.8333173156 \\
\hline $\mathrm{C}$ & -3.6609692226 & 2.9740795831 & 2.0618623747 \\
\hline $\mathrm{C}$ & -1.2389978875 & 3.6963463503 & 2.1998199386 \\
\hline $\mathrm{C}$ & -6.0585251864 & 2.1827053493 & 2.0614101756 \\
\hline $\mathrm{O}$ & 0.463051785 & -1.0178843695 & -1.5664460774 \\
\hline $\mathrm{N}$ & 1.0504618397 & -2.260726747 & 1.4721766211 \\
\hline $\mathrm{N}$ & -0.9106105557 & -2.6704114258 & 0.6548554073 \\
\hline $\mathrm{C}$ & 2.4495383319 & -0.8815578436 & 2.9134927224 \\
\hline $\mathrm{C}$ & 2.3146095442 & -1.6315135662 & 1.7353428179 \\
\hline $\mathrm{C}$ & 0.2450448816 & -0.679503404 & -0.3618098371 \\
\hline $\mathrm{C}$ & 0.1215176715 & -1.8101130599 & 0.6133145717 \\
\hline $\mathrm{C}$ & 3.6908508808 & -0.2918672428 & 3.1536246989 \\
\hline $\mathrm{C}$ & 4.577596238 & -1.2006168012 & 1.1129491433 \\
\hline $\mathrm{C}$ & 3.3534370182 & -1.8041752968 & 0.8134102618 \\
\hline $\mathrm{C}$ & 4.7620067089 & -0.4374848697 & 2.2663862028 \\
\hline $\mathrm{C}$ & 0.5950960199 & -3.4221242818 & 2.076175816 \\
\hline $\mathrm{C}$ & -2.147417588 & -2.5003685336 & -0.0561917415 \\
\hline $\mathrm{C}$ & -2.2737755741 & -3.071728524 & -1.3272704581 \\
\hline $\mathrm{C}$ & -3.1604488318 & -1.7471131621 & 0.5555833017 \\
\hline $\mathrm{C}$ & 1.2894336968 & -0.6863016887 & 3.8384863611 \\
\hline $\mathrm{C}$ & -0.6366753095 & -3.6770140169 & 1.5656514964 \\
\hline $\mathrm{C}$ & 3.1522008787 & -2.5644237818 & -0.4602290205 \\
\hline $\mathrm{C}$ & 6.0664712394 & 0.2518227943 & 2.532223822 \\
\hline $\mathrm{C}$ & -4.5363861836 & -2.1630475915 & -1.4213264428 \\
\hline $\mathrm{C}$ & -4.3563140722 & -1.606898714 & -0.1516640957 \\
\hline $\mathrm{C}$ & -2.9493977127 & -1.0888316186 & 1.8811667764 \\
\hline
\end{tabular}

C $\quad-3.4881598927$

C -1.1283301435

C -5.8216949116

H 3.8145079137

H 5.0961095088

H 0.954797738

H $\quad 0.395876287$

H 1.5956433725

H $\quad 0.8417459134$

H -1.3170405504

H $\quad 2.2172685292$

H 3.6857980605

H 2.1046624953

H 5.9311338802

H 6.7104380202

H $\quad 6.3132595244$

H $\quad-5.3242057145$

H -2.4956184191

H $\quad-3.9523916057$

H -2.3547273727

H -3.815198605

H -0.4783810322

H -1.5348915004

H $\quad-0.7684694142$

H -6.8856281869

H -6.1620032565

H $\quad-6.213967806$

H 3.821896293

H 5.4112147866

H 1.2025990483

H 0.4575184746

H 1.5668559677

H 0.9246353549

H -1.3546541632

H 2.6639750616

H 4.1162548079

H 2.4916409332

H 5.9891596649

H 6.8768231491

H 6.3786997022

H $\quad-5.1744241243$

H $\quad-2.6328972503$

H -3.877653251

H -2.1407257317

H $\quad-3.6139881665$

H $\quad-0.2763621875$

H -1.407210772

H -0.7814387566

H $\quad-6.6977839157$
$-2.8884056761$ $-3.8044317046$ $-1.945762211$ $-0.2418643412$ 1.2268483152 3.7707254169 0.0817307208 0.0571238309 1.5980126865 4.5892754852 3.302688649 2.5699396369 1.7403999401 $-1.2889257872$ 0.2242944888 $-0.0301830604$ 1.3248398767 1.9573772044 0.9460218843 0.3802565358 3.3732443564 2.8974063015 4.0794887079 4.5244108665 2.4190276257 2.8327867546 1.1381348734 0.3096983433

$-1.3229326403$ $-3.9520892252$ $-0.2160588661$ $-0.0313012093$ $-1.6428562594$ $-4.4739856189$ $-3.5441720812$ $-2.7475910005$ $-2.0009161326$ 1.3391002819 $-0.1446185948$ 0.1468230313 $-1.0370482499$ $-1.8143209777$ $-0.5997578012$ $-0.3175135354$ $-3.3242069487$ $-3.1033826279$ $-4.2107449086$ $-4.647997122$ $-2.0500834263$
$-1.9933575615$ $-1.9527994479$ $-2.1620171725$ $-4.1778222649$ $-0.3379517608$ $-2.9241025326$ $-3.5880763061$ $-4.9286100992$ $-4.4318417726$ $-1.5073848646$ 0.2739621518 0.9890619915 1.1117797274 $-2.3403345902$ $-1.808730578$ $-3.534317946$ $-0.4036564964$ $-2.5408789305$ $-2.2986912138$ $-1.7316426341$ 3.0738010255

2.3230120542

3.1921764981

1.6347610854 1.365996534

2.9484031733 2.3998272023 4.0638176771

0.4076412658 2.8096442288 3.2715099548 4.6827108374 4.2622462072 1.758909044 $-0.2863874555$ $-0.9672176599$ $-1.1545008413$ 2.32947106 1.8939775335 3.5879763942 0.310706951 2.6577022418 2.2280962602 1.8206223061 $-2.9937481227$ $-2.0786415154$ $-2.9404746496$ $-1.3237614722$ $-1.4954176321$ 
$\mathbf{C l}_{2}\left[E^{\circ}=-920.0361141 ; G^{\circ}=-920.056732 ; G^{\circ}(T H F)=-920.0599524 ; G^{\circ}(M e C N)=-920.059756\right]$ Def2-SV(P)

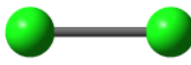

Charge $=0 ;$ Multiplicity $=1$;

\begin{tabular}{llll}
\hline \multicolumn{3}{c}{ Atomic coordinates (Cartesian; in atomic unit) } \\
\hline $\mathrm{Cl}$ & -0.2453610738 & 0.35539215 & 0.0 \\
$\mathrm{Cl}$ & -2.2738546062 & 0.35539215 & 0.0
\end{tabular}

$\mathbf{2}^{2+} \cdot \mathbf{C l}^{-}\left[E^{\circ}=-2531.7803883 ; \mathrm{G}^{\circ}=-2531.050256 ; \mathrm{G}^{\circ}(\mathrm{THF})=-2531.1382281 ;\right.$
$\left.\mathrm{G}^{\circ}(\mathrm{MeCN})=-2531.1482169\right] \operatorname{Def} 2-\mathrm{SV}(\mathrm{P})$

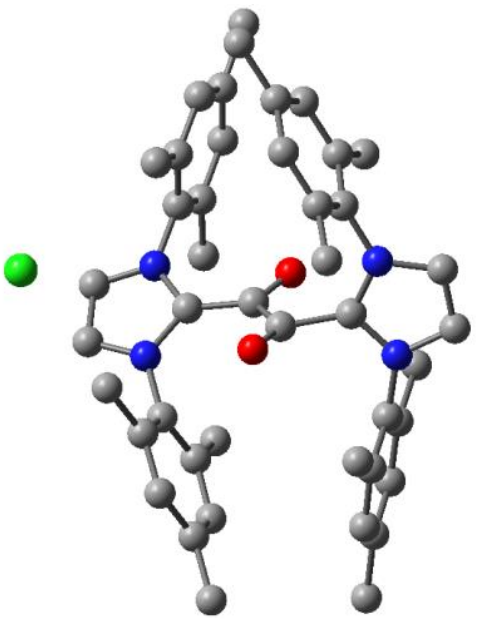

Charge $=1$; Multiplicity $=1$;

\begin{tabular}{llll}
\hline \multicolumn{4}{c}{ Atomic coordinates (Cartesian; in atomic unit) } \\
\hline $\mathrm{O}$ & 0.9268901987 & 1.2766962929 & 1.1411890864 \\
$\mathrm{~N}$ & 0.4105269457 & -1.7430146401 & 1.8834443309 \\
$\mathrm{~N}$ & -1.4601804943 & -2.2356057109 & 0.834314626 \\
$\mathrm{O}$ & -0.6436520587 & -0.5404002886 & -1.3547868724 \\
$\mathrm{~N}$ & 1.6704353021 & 1.8511214516 & -1.9624157885 \\
$\mathrm{~N}$ & -0.2978101819 & 2.6547009038 & -1.520720207 \\
$\mathrm{C}$ & 0.6185821945 & 1.7598039008 & -1.1470612262 \\
$\mathrm{C}$ & -0.3876233945 & -1.3782494808 & 0.8251346579 \\
$\mathrm{C}$ & 2.0920753279 & -0.4985590083 & 3.1689052936 \\
$\mathrm{C}$ & 1.7785322502 & -1.3373707601 & 2.088444782 \\
$\mathrm{C}$ & -2.6255704117 & -2.1303964816 & -0.0143603412 \\
$\mathrm{C}$ & -1.3743223392 & -3.0512797378 & 1.8968222697 \\
$\mathrm{C}$ & -0.1939977357 & -2.7355190415 & 2.5742235465 \\
$\mathrm{C}$ & 0.5312229365 & 0.8705786349 & 0.0790209629 \\
$\mathrm{C}$ & -2.7081338861 & -2.9749085895 & -1.1272722754 \\
$\mathrm{C}$ & -3.6044145759 & -1.1831104956 & 0.3108786723 \\
$\mathrm{C}$ & 3.4322989517 & -0.1438364909 & 3.3221615546 \\
$\mathrm{C}$ & 2.8444203954 & 1.0142771513 & -1.9117462113 \\
$\mathrm{C}$ & 2.7396851193 & -1.8339037309 & 1.1978846714 \\
$\mathrm{C}$ & -1.6270122195 & 3.6702631378 & 0.2523047163 \\
$\mathrm{C}$ & -1.5808155075 & 2.8433828403 & -0.8852699482 \\
$\mathrm{C}$ & -0.1717730264 & -0.4231131616 & -0.2175606746 \\
$\mathrm{C}$ & 2.9119081289 & -0.0670849658 & -2.8083092988 \\
$\mathrm{C}$ & 1.4178044432 & 2.8415698743 & -2.8957648002 \\
$\mathrm{C}$ & -2.863294224 & 3.8312635888 & 0.8709903215 \\
$\mathrm{C}$ & 3.8684645987 & 1.3332070606 & -1.0115482266
\end{tabular}

$\begin{array}{llll}\text { C } & 4.067036623 & -1.4522278548 & 1.4104296221 \\ \text { C } & -2.7105293916 & 2.2211069047 & -1.4319712463 \\ \text { C } & 0.1851130113 & 3.3455149696 & -2.6171547668 \\ \text { C } & -3.8439156725 & -2.8684800943 & -1.9282041008 \\ \text { C } & -4.7233691972 & -1.1267094106 & -0.525368182 \\ \text { C } & -4.8663313227 & -1.9579846577 & -1.6389427964 \\ \text { C } & -3.453587506 & -0.2398658517 & 1.4642916352 \\ \text { C } & 5.1206813112 & -0.5705205725 & -1.895023453 \\ \text { C } & -3.9256777869 & 2.43722719 & -0.773492119 \\ \text { C } & 2.3811450264 & -2.7354936043 & 0.0524437533 \\ \text { C } & 5.0043106386 & 0.5190625306 & -1.0320490141 \\ \text { C } & 1.041341531 & 0.0120002724 & 4.0998818276 \\ \text { C } & -6.0969808777 & -1.8917127634 & -2.4898667658 \\ \text { C } & -4.0233318366 & 3.2206361394 & 0.3755445735 \\ \text { C } & 4.0626935668 & -0.8504226068 & -2.7694662984 \\ \text { C } & 4.4313866705 & -0.6050316562 & 2.4577301328 \\ \text { C } & 1.7944806407 & -0.3630967474 & -3.7622825745 \\ \text { C } & -1.5971634101 & -3.9213936275 & -1.4604539551 \\ \text { C } & -2.6734209928 & 1.3330601984 & -2.6375846692 \\ \text { C } & -0.3969286754 & 4.3530478972 & 0.7702443087 \\ \text { C } & -5.334794207 & 3.4199940201 & 1.0688731221 \\ \text { C } & 3.7626071674 & 2.4570141548 & -0.0277397167 \\ \text { C } & 6.3434157665 & -1.4346736332 & -1.8959882956 \\ \text { C } & 5.8563945087 & -0.1892675853 & 2.6600499226 \\ \text { H } & -2.1494808328 & -3.7760840442 & 2.1434004486 \\ \text { H } & 0.3064018219 & -3.249621887 & 3.3937549733 \\ \text { H } & 3.7073740847 & 0.5149183306 & 4.1569214253\end{array}$


H 2.1487772185

H $\quad-2.928642527$

H 4.8427304329

H -0.404310493

H -3.9341779026

H $\quad-5.5203793705$

H $\quad-3.0729629272$

H -4.4223922108

H $\quad-2.7494128517$

H -4.8287036032

H $\quad 1.5824346859$

H 3.2608475797

H 2.02656009

H 5.8260781599

H $\quad 0.2233788534$

H 1.4707462606

H $\quad 0.5468586839$

H -5.8927901511

H $\quad-6.5266879905$

H -6.8840402925

H 4.1420439965

H $\quad 0.8122972892$

H 1.9580076617

H 1.717313715
3.0885818698

4.461806477

$-1.8470584136$

4.1316623993

$-3.5183828588$

$-0.4096620228$

$-0.7276629651$

0.2320219901

0.5839624745

1.9645789616

$-3.4573280912$

$-3.31592135$

$-2.1631179646$

0.7485337638

0.511499763

0.732589268

$-0.7953433985$

$-2.2013974464$

$-0.87395932$

$-2.5661977208$

$-1.7074426958$

$-0.4057331127$

$-1.3297162821$

0.4068104452
$-3.6659337286$

1.767454346

0.7409777887

$-3.0896900355$

$-2.8085985385$

$-0.283849915$

2.3848420096

1.7060521048

1.2168249708

$-1.1833010532$

0.3127508357

$-0.2781226899$

$-0.8306466067$

$-0.3399874265$

3.547455365

4.8163003922

4.672907653

$-3.5300335292$

$-2.5086078675$

$-2.099836506$

$-3.4519208311$

$-3.2515279531$

$-4.2680782492$

$-4.5553250817$

\section{H $\quad-0.6512634267$}

H -1.8248894024

H $\quad-1.4079498094$

H -1.7488948135

H $\quad-3.5356205538$

H $\quad-2.7368217864$

H $\quad 0.3521389044$

H $\quad-0.6469550458$

H 0.0975842452

H $\quad-5.2397674044$

H -6.109694745

H $\quad-5.7081256645$

H 3.1116392042

H 4.7578202417

H 3.3422141308

H 7.0311343564

H $\quad 6.9065250817$

H $\quad 6.0781569337$

H 5.9848635179

H 6.541904199

H $\quad 6.1923355842$

Cl -1.8913614512
$-3.3700676375$

$-4.4952017493$

$-4.6514517049$

1.4353671992

1.5426985227

0.269506405

3.6287002676

5.0310072468

4.9594099743

3.2608843704

2.7320876599

4.4525596676

3.2801804231

2.8828813573

2.0941573745

$-1.178517211$

$-1.3296682483$

$-2.5051678183$

0.8980216083

$-0.7213577306$

$-0.386847377$

$-1.8966666332$
$-1.6328325385$

$-2.3744529833$

$-0.6496130622$

$-3.2326681621$

$-3.2965469275$

$-2.3334061051$

1.1466823983

1.603188454

$-0.0139553703$

2.1588820144

0.687368759

0.9284954603

$-0.3774413737$

0.1893872352

0.9326174208

$-1.0710889902$

$-2.8429722185$

$-1.8038757158$

2.4903233404

1.9764887115

3.694899357

4.3199934294

\section{Molecular orbitals}
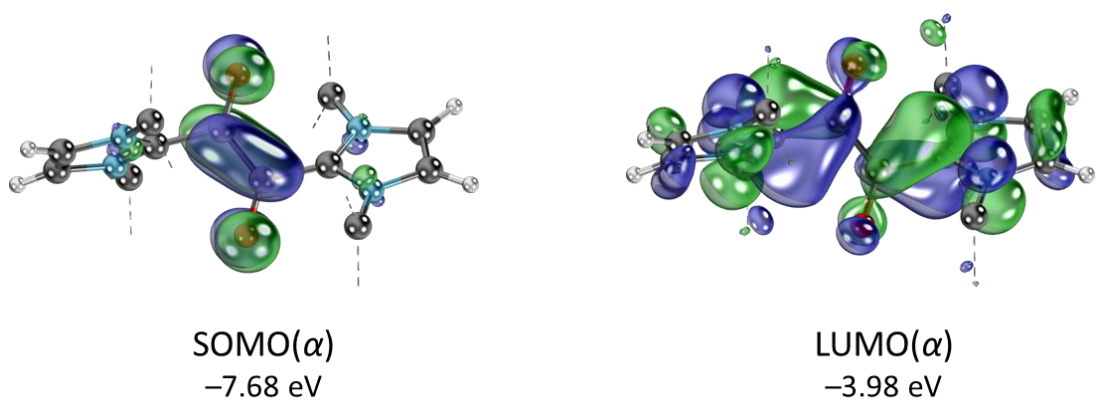

$\operatorname{LUMO}(\alpha)$

$-3.98 \mathrm{eV}$

Figure S5. SOMO and LUMO of $\mathbf{2 a}^{\mathbf{}^{+}}$.

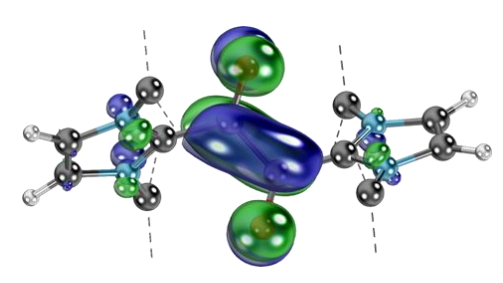

LUMO

$-9.39 \mathrm{eV}$

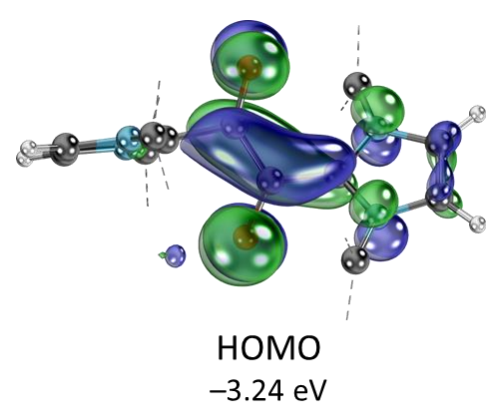

$-3.24 \mathrm{eV}$

Figure S6. LUMO of $\mathbf{2 a}^{\mathbf{2}}$ (left) and HOMO of $\mathbf{2 a}^{\mathbf{0}}$ (right). 
Wiberg bond index

(a)

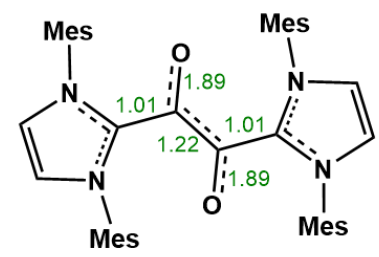

(c)

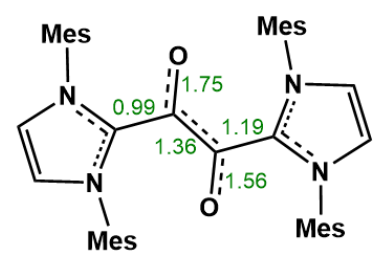

(b)

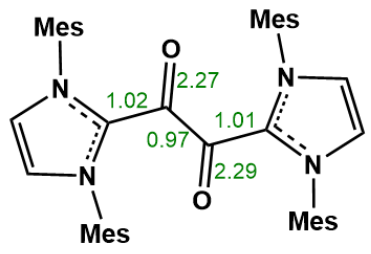

Figure S7. Selected Wiberg bond indices for (a) $\mathbf{2} \mathbf{a}^{\mathbf{}}$, (b) $\mathbf{2} \mathbf{a}^{\mathbf{2 +}}$, and (c) $\mathbf{2} \mathbf{a}^{\mathbf{0}}$. 


\section{X-ray Crystallography}

CCDC 2011174-2011177 and 2050164 contains the supplementary crystallographic data for $\left[\mathbf{2 a}^{\mathbf{*}}\right]\left[\mathrm{BF}_{4}^{-}\right],\left[\mathbf{2 b}^{\mathbf{0}}\right]\left[\mathrm{BF}_{4}^{-}\right],\left[\mathbf{2 a}^{2+}\right]\left[\mathrm{BF}_{4}^{-}\right] 2,\left[\mathbf{2 a}^{\mathbf{}}\right]\left[\mathrm{Cl}^{-}\right]$, and $\mathbf{2 \mathbf { a } ^ { 0 }}$ respectively. These data can be obtained free of charge via https://www.ccdc.cam.ac.uk/

\section{General information}

A suitable crystal was coated with paratone- $N$ oil and the diffraction data measured either with a graphite-monochromated Mo K $\alpha$ radiation source on a Bruker Venture CMOS diffractometer or a synchrotron radiation on a 2D beamline at the Pohang Accelerator Laboratory, Korea. Using Olex2, ${ }^{[10]}$ The structure was solved by ShelXT ${ }^{[11]}$ using intrinsic phasing and refined by ShelXL ${ }^{[12]}$ using least squares minimization. All the non-hydrogen atoms were refined anisotropically. All hydrogen atoms were added to their geometrically ideal positions. Solvent mask ${ }^{[13]}$ was used to exclude solvent and some anion molecules during the refinement of $\left[\mathbf{2 a}^{\mathbf{}}\right]\left[\mathrm{Cl}^{-}\right]($Grid $=0.25 \AA$, Solvent $\mathrm{R}=1.2 \AA$ ).

\begin{tabular}{|c|c|c|c|c|c|}
\hline & {$\left[\mathbf{2 a}^{-+}\right]\left[\mathrm{BF}_{4}^{-}\right]$} & {$\left[2 \mathbf{b}^{\bullet+}\right]\left[\mathrm{BF}_{4}^{-}\right]$} & {$\left[\mathbf{2 a}^{\mathbf{2 +}}\right]\left[\mathrm{BF}_{4}^{-}\right]_{2}$} & {$\left[2 \mathbf{a}^{\bullet+}\right]\left[\mathrm{Cl}^{-}\right]$} & $2 \mathbf{a}^{0}$ \\
\hline Empirical formula & $\mathrm{C}_{44} \mathrm{H}_{48} \mathrm{BF}_{4} \mathrm{~N}_{4} \mathrm{O}_{2}$ & $\mathrm{C}_{56} \mathrm{H}_{72} \mathrm{BF}_{4} \mathrm{~N}_{4} \mathrm{O}_{2}$ & $\mathrm{C}_{46} \mathrm{H}_{52} \mathrm{~B}_{2} \mathrm{Cl}_{4} \mathrm{~F}_{8} \mathrm{~N}_{4} \mathrm{O}_{2}$ & $\mathrm{C}_{44} \mathrm{H}_{48} \mathrm{Cl}_{0.67} \mathrm{~N}_{4} \mathrm{O}_{2}$ & $\mathrm{C}_{44} \mathrm{H}_{48} \mathrm{~N}_{4} \mathrm{O}_{2}$ \\
\hline Formula weight & 751.67 & 919.98 & 1008.33 & 688.49 & 664.86 \\
\hline Temperature/K & 100 & 100 & 100.0 & 293(2) & 100 \\
\hline Crystal system & monoclinic & monoclinic & triclinic & trigonal & orthorhombic \\
\hline Space group & $\mathrm{I} 2 / \mathrm{a}$ & $\mathrm{P} 2_{1} / \mathrm{n}$ & P-1 & P-3c1 & Pbca \\
\hline $\mathrm{a} / \AA$ & $15.4206(9)$ & $14.6496(10)$ & $11.438(7)$ & $20.645(3)$ & $17.113(6)$ \\
\hline $\mathrm{b} / \AA ̊$ & $16.1466(10)$ & $22.7009(14)$ & $11.822(6)$ & $20.645(3)$ & $18.399(6)$ \\
\hline $\mathrm{c} / \AA$ & $17.1235(13)$ & $16.5084(11)$ & $20.180(12)$ & $16.312(3)$ & $22.754(6)$ \\
\hline$\alpha /^{\circ}$ & 90 & 90 & $88.103(15)$ & 90 & 90 \\
\hline$\beta /{ }^{\circ}$ & 108.011(4) & $92.586(2)$ & $84.082(18)$ & 90 & 90 \\
\hline$\gamma /{ }^{\circ}$ & 90 & 90 & $69.483(14)$ & 120 & 90 \\
\hline Volume $/ \AA^{3}$ & $4054.7(5)$ & $5484.4(6)$ & $2542(3)$ & 6021(2) & 7164(4) \\
\hline $\mathrm{Z}$ & 4 & 4 & 2 & 6 & 8 \\
\hline$\rho_{\text {calc }} \mathrm{g} / \mathrm{cm}^{3}$ & 1.231 & 1.114 & 1.317 & 1.139 & 1.233 \\
\hline$\mu / \mathrm{mm}^{-1}$ & 0.088 & 0.076 & 0.302 & 0.111 & 0.076 \\
\hline $\mathrm{F}(000)$ & 1588.0 & 1972.0 & 1044.0 & 2204.0 & 2848.0 \\
\hline Crystal size $/ \mathrm{mm}^{3}$ & $0.15 \times 0.08 \times 0.05$ & $0.3 \times 0.05 \times 0.05$ & $0.3 \times 0.15 \times 0.03$ & $0.08 \times 0.04 \times 0.03$ & $0.06 \times 0.05 \times 0.02$ \\
\hline Radiation & $\begin{array}{l}\operatorname{MoK} \alpha \\
(\lambda=0.71073)\end{array}$ & $\begin{array}{l}\operatorname{MoK} \alpha \\
(\lambda=0.71073)\end{array}$ & $\begin{array}{l}\operatorname{MoK} \alpha \\
(\lambda=0.71073)\end{array}$ & $\begin{array}{l}\text { synchrotron } \\
(\lambda=0.700)\end{array}$ & $\begin{array}{l}\operatorname{MoK} \alpha \\
(\lambda=0.71073)\end{array}$ \\
\hline $\begin{array}{l}2 \Theta \text { range for } \\
\text { data collection } /{ }^{\circ}\end{array}$ & 5.046 to 52.178 & 5.11 to 50.066 & 4.28 to 50.054 & 3.886 to 59.632 & 3.58 to 50.052 \\
\hline Index ranges & $\begin{array}{l}-19 \leq \mathrm{h} \leq 19 \\
-19 \leq \mathrm{k} \leq 19 \\
-21 \leq 1 \leq 21\end{array}$ & $\begin{array}{l}-17 \leq \mathrm{h} \leq 17 \\
-27 \leq \mathrm{k} \leq 27 \\
-19 \leq 1 \leq 19\end{array}$ & $\begin{array}{l}-13 \leq \mathrm{h} \leq 13 \\
-14 \leq \mathrm{k} \leq 13 \\
-24 \leq 1 \leq 24\end{array}$ & $\begin{array}{l}-29 \leq \mathrm{h} \leq 29 \\
-24 \leq \mathrm{k} \leq 24 \\
-20 \leq 1 \leq 17\end{array}$ & $\begin{array}{l}-20 \leq \mathrm{h} \leq 20 \\
-21 \leq \mathrm{k} \leq 21 \\
-27 \leq 1 \leq 27\end{array}$ \\
\hline Reflections collected & 33904 & 84766 & 31408 & 15034 & 153360 \\
\hline $\begin{array}{l}\text { Independent } \\
\text { reflections }\end{array}$ & $\begin{array}{l}3995\left[R_{\text {int }}=0.0645,\right. \\
\left.R_{\text {sigma }}=0.0298\right]\end{array}$ & $\begin{array}{l}9692\left[\mathrm{R}_{\text {int }}=0.1212,\right. \\
\left.\mathrm{R}_{\text {sigma }}=0.0610\right]\end{array}$ & $\begin{array}{l}8929\left[\mathrm{R}_{\text {int }}=0.0963,\right. \\
\left.\mathrm{R}_{\text {sigma }}=0.0907\right]\end{array}$ & $\begin{array}{l}4642\left[\mathrm{R}_{\text {int }}=0.0421,\right. \\
\left.\mathrm{R}_{\text {sigma }}=0.0458\right]\end{array}$ & $\begin{array}{l}6320\left[\mathrm{R}_{\text {int }}=0.1383,\right. \\
\left.\mathrm{R}_{\text {sigma }}=0.0527\right]\end{array}$ \\
\hline $\begin{array}{l}\text { Data/restraints } \\
\text { /parameters }\end{array}$ & $3995 / 0 / 255$ & $9692 / 60 / 648$ & $8929 / 78 / 644$ & $4642 / 0 / 235$ & $6320 / 0 / 463$ \\
\hline Goodness-of-fit on $\mathrm{F}^{2}$ & 1.071 & 1.026 & 1.020 & 1.103 & 1.036 \\
\hline $\begin{array}{l}\text { Final } R \text { indexes } \\
{[I>=2 \sigma(I)]}\end{array}$ & $\begin{array}{l}\mathrm{R}_{1}=0.0561 \\
\mathrm{wR}_{2}=0.1386\end{array}$ & $\begin{array}{l}\mathrm{R}_{1}=0.0898 \\
\mathrm{wR}_{2}=0.2465\end{array}$ & $\begin{array}{l}\mathrm{R}_{1}=0.0847 \\
\mathrm{wR}_{2}=0.1956\end{array}$ & $\begin{array}{l}\mathrm{R}_{1}=0.0596 \\
\mathrm{wR}_{2}=0.1617\end{array}$ & $\begin{array}{l}\mathrm{R}_{1}=0.0545 \\
\mathrm{wR}_{2}=0.1250\end{array}$ \\
\hline $\begin{array}{l}\text { Final } \mathrm{R} \text { indexes } \\
\text { [all data] }\end{array}$ & $\begin{array}{l}\mathrm{R}_{1}=0.0829 \\
\mathrm{wR}_{2}=0.1588\end{array}$ & $\begin{array}{l}\mathrm{R}_{1}=0.1618 \\
\mathrm{wR}_{2}=0.3018\end{array}$ & $\begin{array}{l}\mathrm{R}_{1}=0.1496 \\
\mathrm{wR}_{2}=0.2372\end{array}$ & $\begin{array}{l}\mathrm{R}_{1}=0.0815 \\
\mathrm{wR}_{2}=0.1724\end{array}$ & $\begin{array}{l}\mathrm{R}_{1}=0.0907 \\
\mathrm{wR}_{2}=0.1457\end{array}$ \\
\hline $\begin{array}{l}\text { Largest diff. peak } \\
\text { /hole / e } \AA^{-3}\end{array}$ & $0.22 /-0.23$ & $0.50 /-0.45$ & $0.45 /-0.43$ & $0.36 /-0.62$ & $0.68 /-0.29$ \\
\hline
\end{tabular}




\section{Structural data}

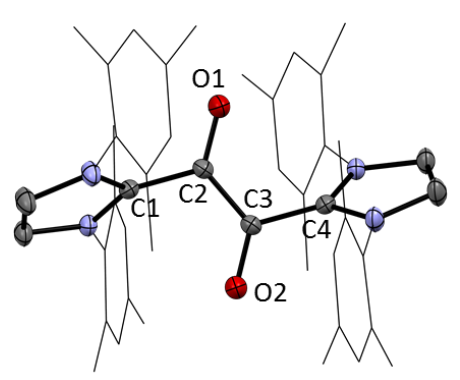

$\left[2 \mathbf{a}^{\cdot+}\right]\left[\mathrm{Cl}^{-}\right]$

Table S1. Selected bond lengths $(\AA)$ and angles $\left({ }^{\circ}\right)$.

\begin{tabular}{|c|c|c|c|c|c|c|}
\hline Bond length $(\AA)$ & $\mathrm{C} 1-\mathrm{C} 2$ & \multicolumn{2}{|c|}{$\mathrm{C} 2-\mathrm{C} 3$} & $\mathrm{C} 3-\mathrm{C} 4$ & $\mathrm{O} 1-\mathrm{C} 2$ & $\mathrm{O} 2-\mathrm{C} 3$ \\
\hline$\left[2 \mathbf{a}^{\mathbf{a}^{+}}\right]\left[\mathrm{BF}_{4}^{-}\right]$ & $1.495(3)$ & \multicolumn{2}{|c|}{$1.409(2)$} & $1.495(3)$ & $1.246(3)$ & $1.246(3)$ \\
\hline$\left[2 \mathbf{b}^{\mathbf{}^{+}}\right]\left[\mathrm{BF}_{4}^{-}\right]$ & $1.497(5)$ & \multicolumn{2}{|c|}{$1.407(4)$} & $1.499(5)$ & $1.247(4)$ & $1.251(4)$ \\
\hline$\left[2 \mathbf{a}^{\cdot+}\right]\left[\mathrm{Cl}^{-}\right]$ & $1.498(2)$ & \multicolumn{2}{|c|}{$1.433(3)$} & $1.498(2)$ & $1.249(3)$ & $1.249(3)$ \\
\hline$\left[2 \mathbf{a}^{2+}\right]\left[\mathrm{BF}_{4}^{-}\right]_{2}$ & $1.488(5)$ & \multicolumn{2}{|c|}{$1.535(5)$} & $1.480(5)$ & $1.190(5)$ & $1.202(5)$ \\
\hline $2 a^{0}$ & $1.510(4)$ & \multicolumn{2}{|c|}{$1.349(4)$} & $1.477(4)$ & $1.300(3)$ & 1.311(3) \\
\hline Bond angle $\left({ }^{\circ}\right)$ & $\mathrm{C} 1-\mathrm{C} 2-\mathrm{O} 1$ & $\mathrm{O} 1-\mathrm{C} 2-\mathrm{C} 3$ & $\mathrm{C} 1-\mathrm{C} 2-\mathrm{C} 3$ & $\mathrm{C} 2-\mathrm{C} 3-\mathrm{O} 2$ & $\mathrm{C} 2-\mathrm{C} 3-\mathrm{C} 4$ & $\mathrm{O} 2-\mathrm{C} 3-\mathrm{C} 4$ \\
\hline$\left[\mathbf{2 a}^{\mathbf{0}}\right]\left[\mathrm{BF}_{4}^{-}\right]$ & $118.2(2)$ & $125.1(2)$ & $116.5(2)$ & $125.1(2)$ & $116.5(2)$ & $118.2(2)$ \\
\hline$\left[2 \mathbf{b}^{\mathbf{}}\right]\left[\mathrm{BF}_{4}^{-}\right]$ & 118.2(3) & $123.5(3)$ & 118.3(3) & 123.5(3) & $117.8(3)$ & 118.5(3) \\
\hline$\left[2 \mathbf{a}^{\cdot+}\right]\left[\mathrm{Cl}^{-}\right]$ & 119.3(2) & $125.2(2)$ & 115.3(2) & $125.2(2)$ & 115.3(2) & 119.3(2) \\
\hline$\left[2 \mathbf{a}^{2+}\right]\left[\mathrm{BF}_{4}^{-}\right]_{2}$ & 121.3(4) & $120.7(4)$ & 117.3(3) & $119.0(4)$ & 119.2(3) & 121.1(4) \\
\hline $2 \mathbf{a}^{0}$ & 117.1(2) & $131.8(3)$ & $110.9(2)$ & $128.8(3)$ & $112.8(2)$ & 118.2(2) \\
\hline Torsion angle $\left(^{\circ}\right)$ & NHC & $-\mathrm{C} 1-\mathrm{C} 2-\mathrm{O} 1$ & $\mathrm{O} 1-$ & $2-\mathrm{C} 3-\mathrm{O} 2$ & $\mathrm{O} 2-\mathrm{C} 3$ & $4-\mathrm{NHC} 2$ \\
\hline$\left[\mathbf{2 a}^{\mathbf{}}\right]\left[\mathrm{BF}_{4}^{-}\right]$ & & $53.0(3)$ & & $2.8(2)$ & & $0(3)$ \\
\hline$\left[2 \mathbf{b}^{\mathbf{}+}\right]\left[\mathrm{BF}_{4}^{-}\right]$ & & $66.9(5)$ & & $9.5(4)$ & & $0(5)$ \\
\hline$\left[2 \mathbf{a}^{\cdot+}\right]\left[\mathrm{Cl}^{-}\right]$ & & $57.8(3)$ & & $1.0(2)$ & & 8(3) \\
\hline$\left[2 \mathbf{a}^{2+}\right]\left[\mathrm{BF}_{4}^{-}\right]_{2}$ & & $44.9(6)$ & & $6.8(4)$ & & 6(6) \\
\hline $2 \mathbf{a}^{0}$ & & $54.7(3)$ & & $0.0(3)$ & & 7(4) \\
\hline
\end{tabular}

The thermal ellipsoids are set at a 50\% probability level. Hydrogens, solvents, disorders were omitted for clarity. 
Table S2. Metric comparison between DFT optimized and X-ray determined structures.

\begin{tabular}{|c|c|c|c|c|c|c|}
\hline Bond length $(\AA)$ & $\mathrm{C} 1-\mathrm{C} 2$ & \multicolumn{2}{|c|}{$\mathrm{C} 2-\mathrm{C} 3$} & $\mathrm{C} 3-\mathrm{C} 4$ & $\mathrm{O} 1-\mathrm{C} 2$ & $\mathrm{O} 2-\mathrm{C} 3$ \\
\hline$\left[\mathbf{2 a}^{\mathbf{*}}\right]\left[\mathrm{BF}_{4}^{-}\right]$(X-ray) & $1.495(3)$ & \multicolumn{2}{|c|}{$1.409(2)$} & $1.495(3)$ & $1.246(3)$ & $1.246(3)$ \\
\hline $\mathbf{2} \mathbf{a}^{\cdot+}(\mathrm{DFT})$ & 1.493 & \multicolumn{2}{|c|}{1.433} & 1.491 & 1.248 & 1.249 \\
\hline$\left[\mathbf{2 a}^{\mathbf{2}}\right]\left[\mathrm{BF}_{4}^{-}\right]_{2}$ (X-ray) & $1.488(5)$ & \multicolumn{2}{|c|}{$1.535(5)$} & $1.480(5)$ & $1.190(5)$ & $1.202(5)$ \\
\hline $\mathbf{2 a}^{2+}(\mathrm{DFT})$ & 1.498 & \multicolumn{2}{|c|}{1.529} & 1.490 & 1.201 & 1.203 \\
\hline $\mathbf{2 a}^{0}$ (X-ray) & $1.510(4)$ & \multicolumn{2}{|c|}{$1.349(4)$} & $1.477(4)$ & $1.300(3)$ & $1.311(3)$ \\
\hline $\mathbf{2 a}^{0}(\mathrm{DFT})$ & 1.498 & \multicolumn{2}{|c|}{1.404} & 1.433 & 1.270 & 1.305 \\
\hline Bond angle $\left(^{\circ}\right)$ & $\mathrm{C} 1-\mathrm{C} 2-\mathrm{O} 1$ & $\mathrm{O} 1-\mathrm{C} 2-\mathrm{C} 3$ & $\mathrm{C} 1-\mathrm{C} 2-\mathrm{C} 3$ & $\mathrm{C} 2-\mathrm{C} 3-\mathrm{O} 2$ & $\mathrm{C} 2-\mathrm{C} 3-\mathrm{C} 4$ & $\mathrm{O} 2-\mathrm{C} 3-\mathrm{C} 4$ \\
\hline$\left[\mathbf{2 a}^{\cdot+}\right]\left[\mathrm{BF}_{4}^{-}\right](\mathrm{X}-\mathrm{ray})$ & $118.2(2)$ & $125.1(2)$ & $116.5(2)$ & $125.1(2)$ & $116.5(2)$ & $118.2(2)$ \\
\hline $\mathbf{2 a}^{\mathbf{a}^{+}}(\mathrm{DFT})$ & 118.5 & 125.8 & 115.6 & 125.5 & 115.8 & 118.5 \\
\hline$\left[\mathbf{2 a}^{\mathbf{2 +}}\right]\left[\mathrm{BF}_{4}^{-}\right]_{2}$ (X-ray) & $121.3(4)$ & $120.7(4)$ & 117.3(3) & $119.0(4)$ & 119.2(3) & $121.1(4)$ \\
\hline $\mathbf{2 a}^{2+}(\mathrm{DFT})$ & 121.6 & 121.5 & 116.2 & 120.5 & 117.4 & 121.4 \\
\hline $2 \mathbf{a}^{0}(X-$ ray $)$ & $117.1(2)$ & $131.8(3)$ & $110.9(2)$ & $128.8(3)$ & $112.8(2)$ & $118.2(2)$ \\
\hline $\mathbf{2 a}^{0}(\mathrm{DFT})$ & 115.5 & 133.0 & 111.2 & 123.5 & 116.7 & 119.6 \\
\hline Torsion angle $\left({ }^{\circ}\right)$ & $\mathrm{NHC}$ & $\mathrm{C}-\mathrm{C} 1-\mathrm{C} 2-\mathrm{O} 1$ & $\mathrm{O} 1-$ & $\mathrm{C} 2-\mathrm{C} 3-\mathrm{O} 2$ & $\mathrm{O} 2-\mathrm{C} 3$ & $4-\mathrm{NHC} 2$ \\
\hline$\left[\mathbf{2 a}^{\mathbf{*}}\right]\left[\mathrm{BF}_{4}^{-}\right]$(X-ray) & & $53.0(3)$ & & $72.8(2)$ & & (3) \\
\hline $\mathbf{2 \mathbf { a } ^ { + + }}(\mathrm{DFT})$ & & 57.5 & & 172.7 & & .1 \\
\hline$\left[\mathbf{2 a}^{\mathbf{2}}\right]\left[\mathrm{BF}_{4}^{-}\right]_{2}(\mathrm{X}$-ray $)$ & & $44.9(6)$ & & $56.8(4)$ & & $6(6)$ \\
\hline $\mathbf{2 a}^{2+}(\mathrm{DFT})$ & & 56.8 & & 165.1 & & .5 \\
\hline $\mathbf{2 a}^{0}(\mathrm{X}$-ray $)$ & & $54.7(3)$ & & $70.0(3)$ & & 7(4) \\
\hline $\mathbf{2 a}^{0}(\mathrm{DFT})$ & & 70.1 & & 173.0 & & 2 \\
\hline
\end{tabular}

DFT calculation at M06/Def2-SV(P). 


\section{EPR}

\section{General information}

EPR spectra were recorded on a Bruker X-band A200 spectrometer using dry dichloromethane solutions. Spectra processing and simulation were performed with Bruker WIN-EPR and EasySpin associated with DFT calculations. ${ }^{[14]}$ Isotropic hyperfine coupling constants were initially computed using gaussian09 with M06/Def2-SV(P) basis sets, and then rescaled to fit the experimental data.

\section{EPR spectra}

(a)

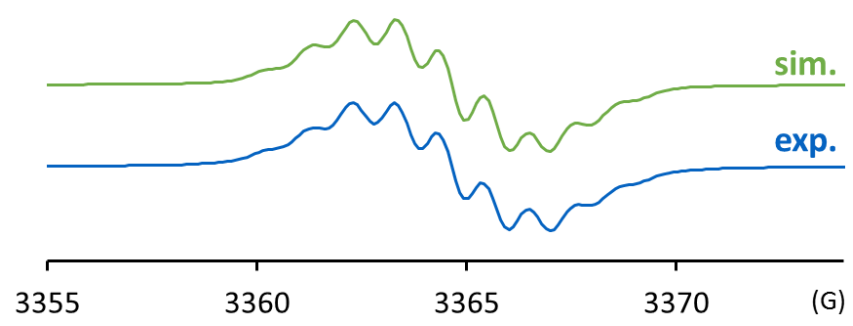

(b)

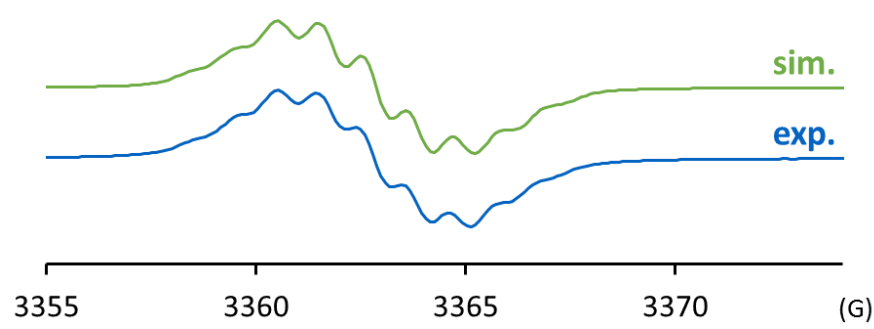

Figure S8. (a) Experimental (bottom, blue) and simulated (top, green) EPR spectra of $\left[\mathbf{2 a}^{\cdot+}\right]\left[\mathbf{B F}_{4}{ }^{-}\right]$ at microwave frequency $=9.4467 \mathrm{GHz}$. Simulated with the following parameters: $g_{\text {iso }}=2.0060$; hyperfine coupling constants: $a\left({ }^{14} \mathrm{~N}\right)=3.0,3.0,2.6,2.6 \mathrm{MHz}$; Gaussian line width $=0.04 \mathrm{mT}$; Lorentzian line width $=0.04 \mathrm{mT}$. (b) Experimental (bottom, blue) and simulated (top, green) EPR spectra of $\left[\mathbf{2 b}^{\mathbf{}}\right]\left[\mathbf{B F}_{4}^{-}\right]$at microwave frequency $=9.4413 \mathrm{GHz}$. Simulated with the following parameters: $g_{\text {iso }}=2.0059$; hyperfine coupling constants: $a\left({ }^{14} \mathrm{~N}\right)=3.0,3.0,2.6,2.6 \mathrm{MHz}$; Gaussian line width $=0.05 \mathrm{mT}$; Lorentzian line width $=0.04 \mathrm{mT}$.

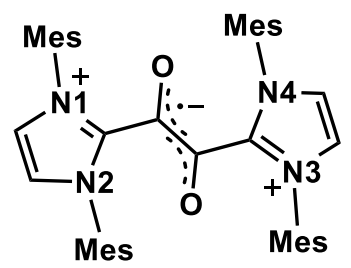

\begin{tabular}{ccccc}
\hline hfcc & N1 & N2 & N3 & N4 \\
\hline $\operatorname{Exp}$ & 2.6 & 3.0 & 2.6 & 3.0 \\
M06/6-31G(d,p) & 2.8 & 3.1 & 2.8 & 3.0 \\
M06/Def2SV(P) & 3.7 & 4.5 & 3.6 & 4.4 \\
\hline
\end{tabular}

Figure S9. Comparison of hyperfine coupling constants for $\left[\mathbf{2 a}^{\mathbf{a}^{+}}\right]\left[\mathbf{B F}_{4}{ }^{-}\right]$from experiment and DFT calculations at M06/6-31G(d,p) or M06/Def2-SV(P). 


\section{Thermal stability}

a)

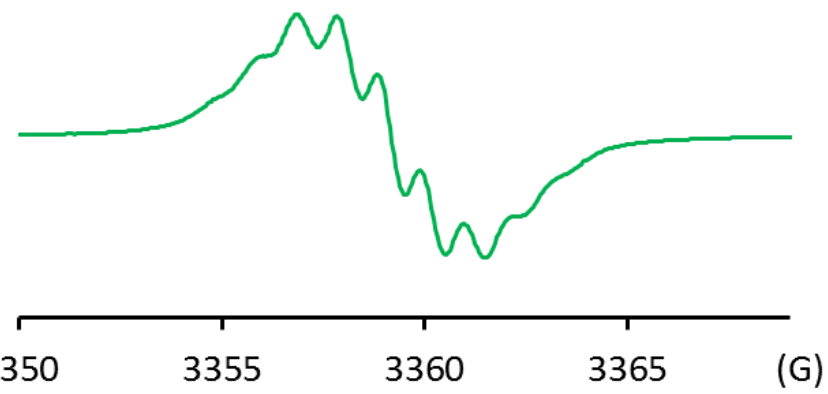

b)

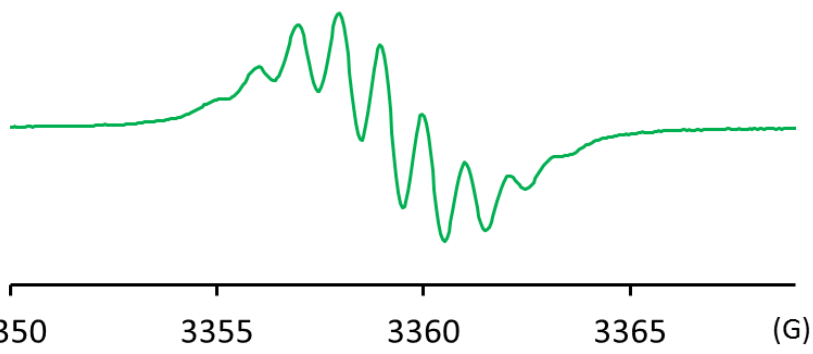

Figure S10. (a) Experimental EPR spectra of $\left[\mathbf{2 a}^{\cdot+}\right]\left[\mathbf{C l}^{-}\right]$after heating for 7 days at $120^{\circ} \mathrm{C}$ under air (in solid state) at microwave frequency $=9.4328 \mathrm{GHz}$ (measured with dichloromethane solution). (b) Experimental EPR spectra of $\left[\mathbf{2 a}^{\circ+}\right]\left[\mathbf{C l}^{-}\right]$after heating for 6 hours at $200^{\circ} \mathrm{C}$ under air (in solid state) at microwave frequency $=9.4337 \mathrm{GHz}$ (measured with dichloromethane solution). 


\section{Cyclic Voltammetry}

\section{General information}

Cyclic voltammograms were recorded at room temperature with either a BioLogic SP-300 potentiostat or Princeton Applied Research (PAR) VersaSTAT 3 potentiostat. The working electrode was a glassy carbon disk (area $\left.=0.02 \mathrm{~cm}^{2}\right)$, the reference electrode was $\mathrm{Ag} / \mathrm{AgCl}$ (saturated), and the counter electrode was a platinum wire.

\section{Cyclic voltammograms}

(a)

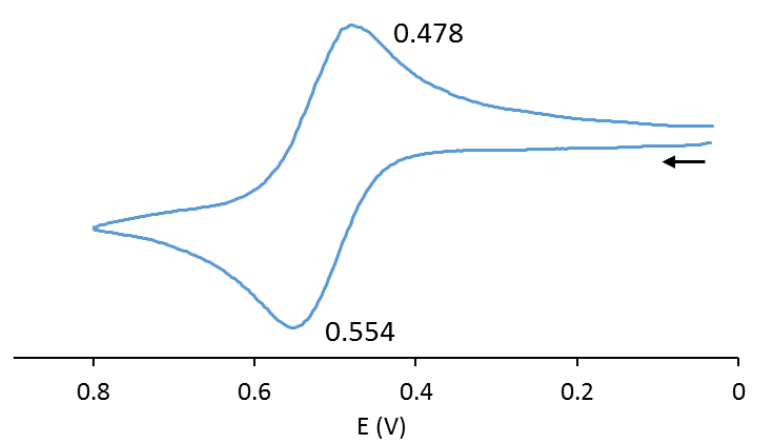

(b)

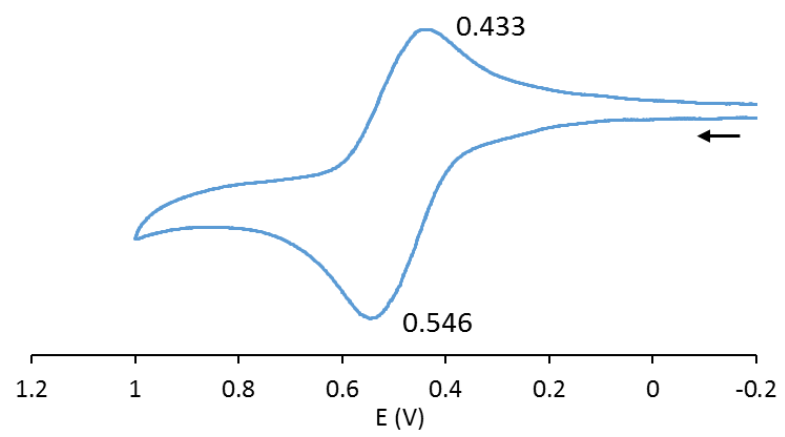

Figure S11. (a) Cyclic voltammogram of $\left[\mathbf{2 a}^{\bullet+}\right]\left[\mathbf{B F}_{4}{ }^{-}\right](3.1 \mathrm{mM})$ in dry and degassed acetonitrile with $\mathrm{Bu}_{4} \mathrm{NPF}_{6}(0.1 \mathrm{M})$ as the supporting electrolyte ( $\mathrm{scan}$ rate $\left.=0.1 \mathrm{~V} / \mathrm{s}\right)$. The cyclic voltammogram showed reversible one-electron redox potential at $\mathrm{E}_{1 / 2}=0.516 \mathrm{~V}$ versus $\mathrm{Ag} / \mathrm{AgCl}$ (saturated)

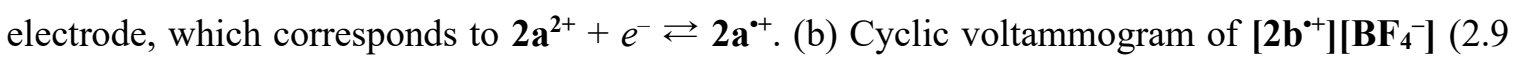
$\mathrm{mM})$ in dry and degassed acetonitrile with $\mathrm{Bu}_{4} \mathrm{NPF}_{6}(0.1 \mathrm{M})$ as the supporting electrolyte (scan rate $=0.1 \mathrm{~V} / \mathrm{s}$ ). The cyclic voltammogram showed reversible one-electron redox potential at $\mathrm{E}_{1 / 2}=0.490$

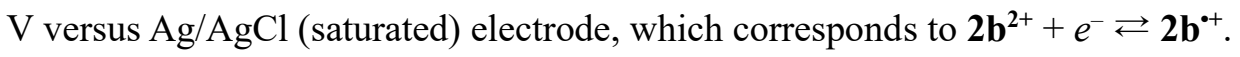




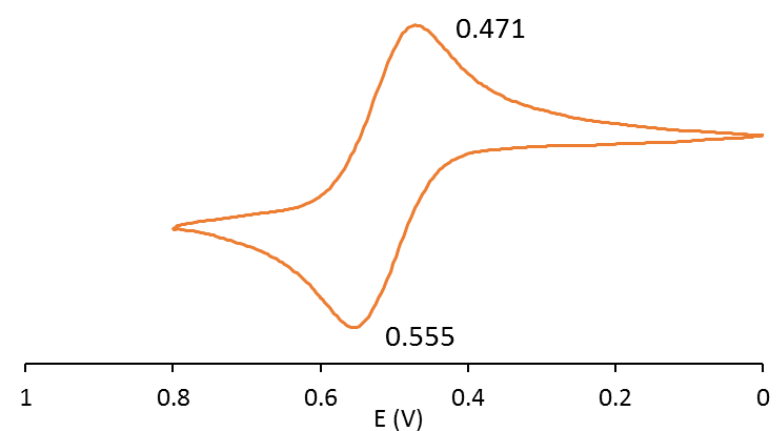

Figure S12. Cyclic voltammogram of $\left[\mathbf{2 a}^{\mathbf{2 +}}\right]\left[\mathbf{B F}_{4}^{-}\right]_{2}(2.6 \mathrm{mM})$ in dry and degassed acetonitrile with $\mathrm{Bu}_{4} \mathrm{NPF}_{6}(0.1 \mathrm{M})$ as the supporting electrolyte $($ scan rate $=0.1 \mathrm{~V} / \mathrm{s})$. The cyclic voltammogram was identical to that of $\left[\mathbf{2 a}^{\cdot+}\right]\left[\mathbf{B F}_{4}{ }^{-}\right]$, showing reversible one-electron redox potential at $\mathrm{E}_{1 / 2}=0.513 \mathrm{~V}$ versus $\mathrm{Ag} / \mathrm{AgCl}$ (saturated) electrode, which corresponds to $\mathbf{2 a}^{2+}+e^{-} \rightleftarrows \mathbf{2 a}^{\mathbf{0}^{++}}$.

(a)

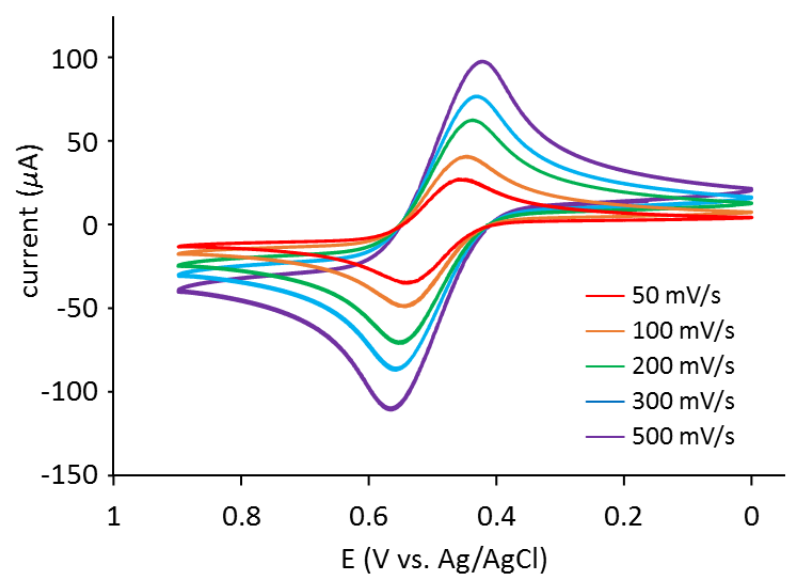

(b)

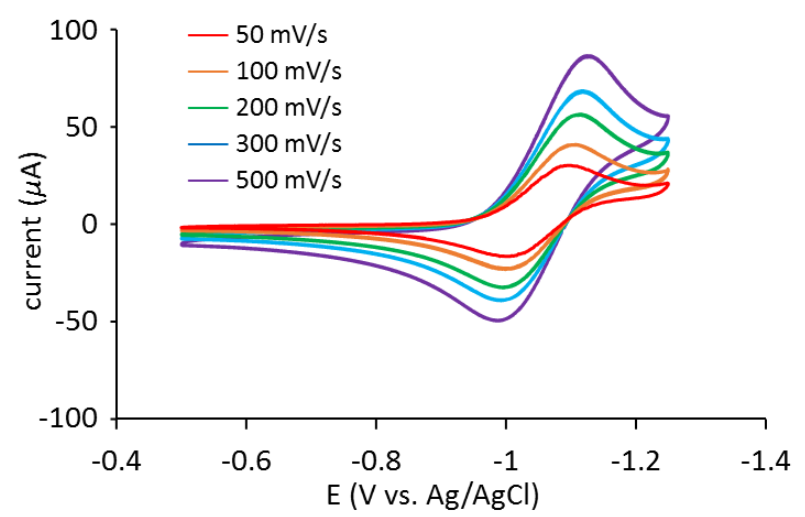

Figure S13. Cyclic voltammogram of $\left[\mathbf{2 a}^{\circ+}\right]\left[\mathbf{B F}_{4}^{-}\right](4.5 \mathrm{mM})$ in dry and degassed acetonitrile with $\mathrm{Bu}_{4} \mathrm{NPF}_{6}(0.1 \mathrm{M})$ as the supporting electrolyte under various scan rates. (a) Oxidative potential scan showing reversible one-electron redox potential at $\mathrm{E}_{1 / 2}=0.498 \mathrm{~V}$ versus $\mathrm{Ag} / \mathrm{AgCl}$ (saturated) electrode, which corresponds to $\mathbf{2 a}^{\mathbf{2 +}}+e^{-} \rightleftarrows \mathbf{2 a}^{\mathbf{*}}$. (a) Reductive potential scan showing reversible one-electron redox potential at $\mathrm{E}_{1 / 2}=-1.051 \mathrm{~V}$ versus $\mathrm{Ag} / \mathrm{AgCl}$ (saturated) electrode, which 
corresponds to $\mathbf{2 a}^{\cdot+}+e^{-} \rightleftarrows \mathbf{2 a}^{\mathbf{0}}$.

(a)

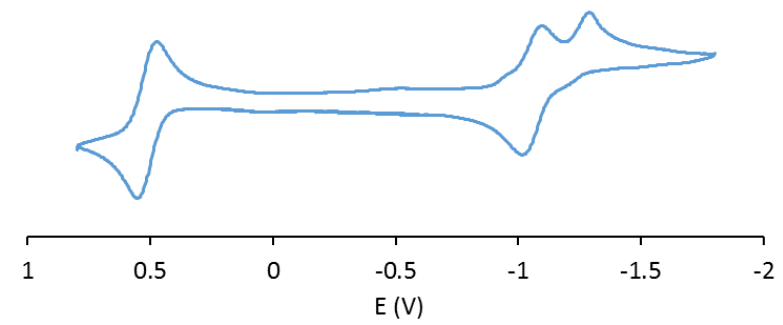

(b)

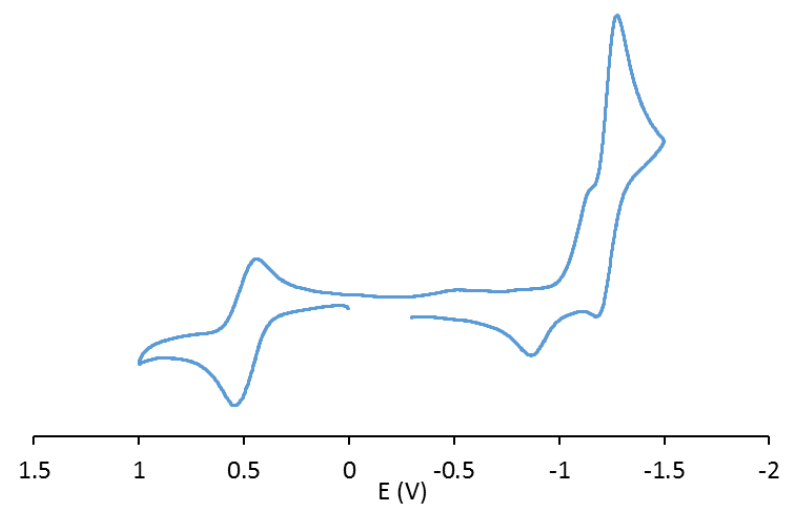

Figure S14. Extended range scan of (a) $\left[\mathbf{2 a}^{\mathbf{2 +}}\right]\left[\mathbf{B F}_{4}^{-}\right]_{2}(2.6 \mathrm{mM})$ and (b) $\left[\mathbf{2} \mathbf{b}^{\text {++}}\right]\left[\mathbf{B F}_{4}^{-}\right](2.9 \mathrm{mM})$ in dry and degassed acetonitrile $\left(0.1 \mathrm{M} \mathrm{Bu}_{4} \mathrm{NPF}_{6}\right.$; scan rate $\left.=0.1 \mathrm{~V} / \mathrm{s}\right)$. Potential versus $\mathrm{Ag} / \mathrm{AgCl}$ (saturated) electrode. 


\section{UV-Vis Spectroscopy}

\section{General information}

The UV-vis spectra were recorded at room temperature with Cary 6000i UV-Vis-NIR (Agilent Technologies) with quartz UV cell.

\section{Experimental and simulated UV-Vis spectra}

(a)

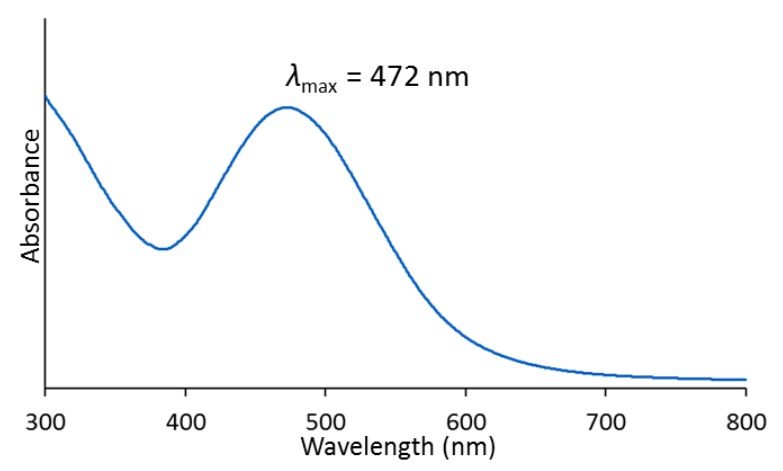

(b)

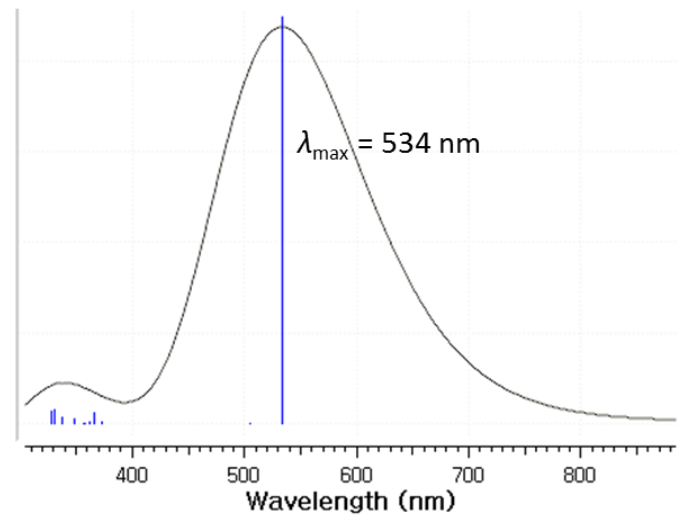

Figure S15. (a) UV-vis absorption of $\left[\mathbf{2 a}^{\cdot+}\right]\left[\mathbf{C l}^{-}\right]$in distilled water (path length $=10 \mathrm{~mm}$ ). (b) Simulated UV-vis absorption from DFT calculation at M06/Def2-SV(P).

(a)

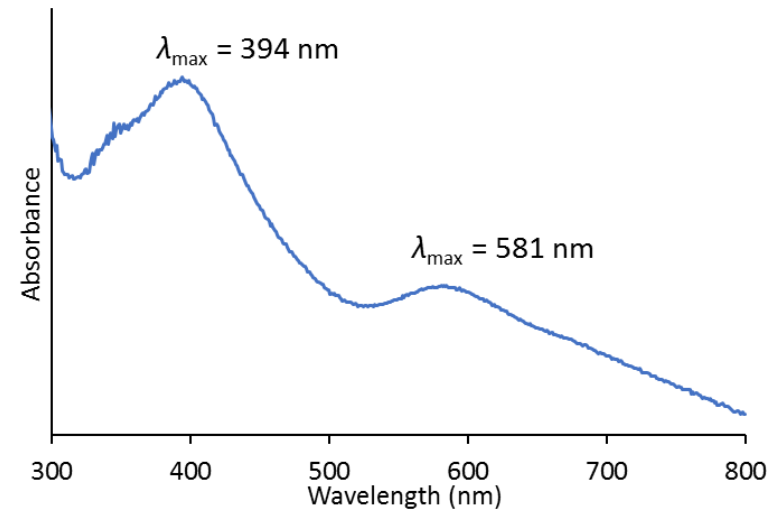

(b)

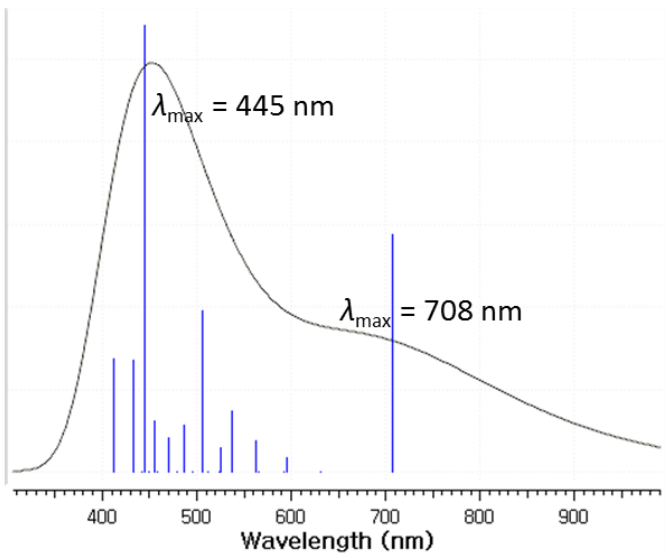

Figure S16. (a) UV-vis absorption of $\mathbf{2} \mathbf{a}^{\mathbf{0}}$ in dry benzene (path length $=1 \mathrm{~mm}$ ). (b) Simulated UVvis absorption from DFT calculation at M06/Def2-SV(P). 


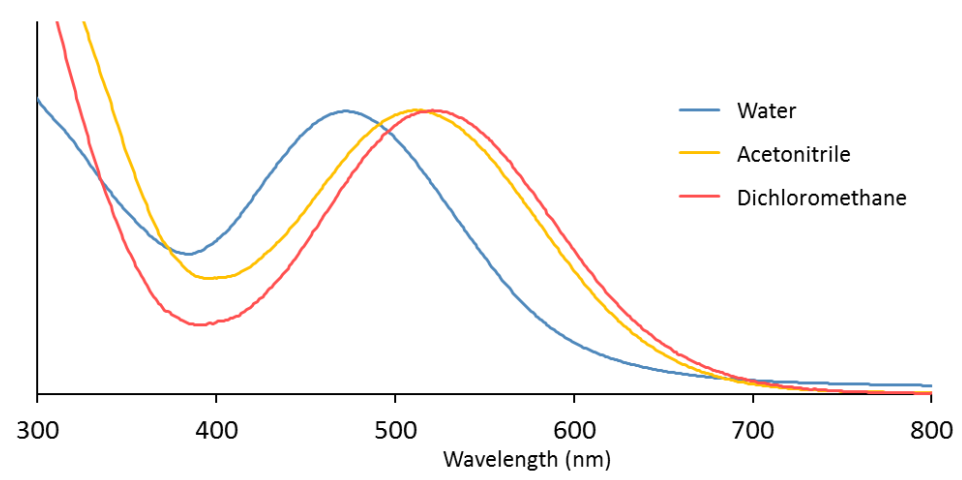

Figure S17. Comparison of UV-vis spectra of $\left[\mathbf{2 a}^{\cdot+}\right]\left[\mathbf{C l}^{-}\right]$in distilled water (blue; $\lambda_{\max }=472 \mathrm{~nm}$ ), acetonitrile (yellow; $\lambda_{\max }=510 \mathrm{~nm}$ ), and dichloromethane (red; $\lambda_{\max }=521 \mathrm{~nm}$ ).

\section{Stability}

(a)

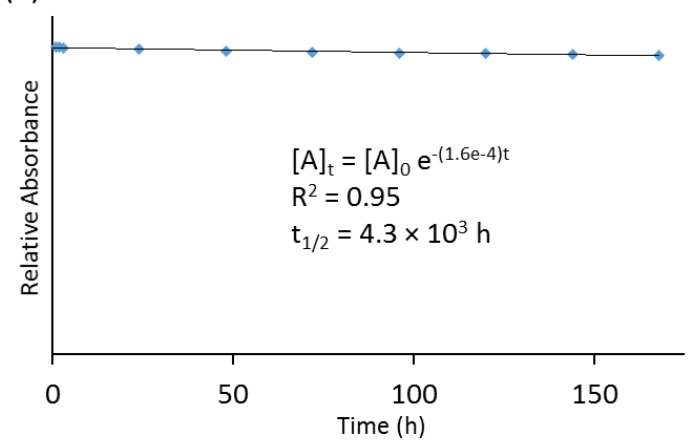

(b)

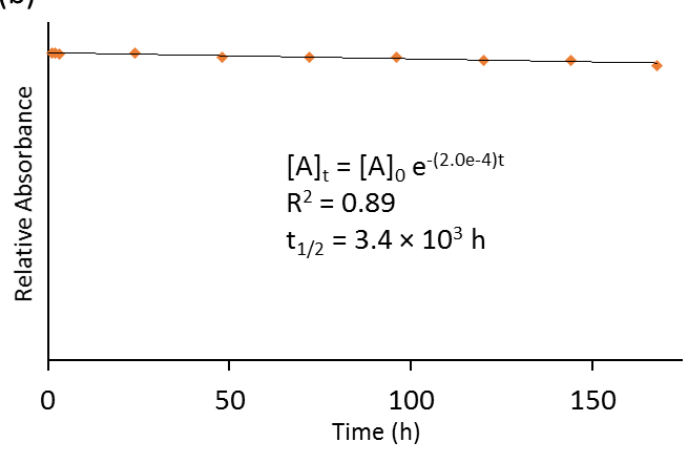

Figure S18. Decay of $\left[\mathbf{2 a}^{\cdot+}\right]\left[\mathrm{Cl}^{-}\right]\left(0.16 \mathrm{mM}\right.$; peak height at $\left.\lambda_{\max }=472 \mathrm{~nm}\right)$ in $0.1 \mathrm{M}$ potassium phosphate buffer (pH 7.4) (a) without sodium ascorbate and (b) with excess sodium ascorbate (4 $\mathrm{mM}$ ) monitored by UV-vis. Half-life was calculated by assuming $1^{\text {st }}$ order kinetics.

(a)

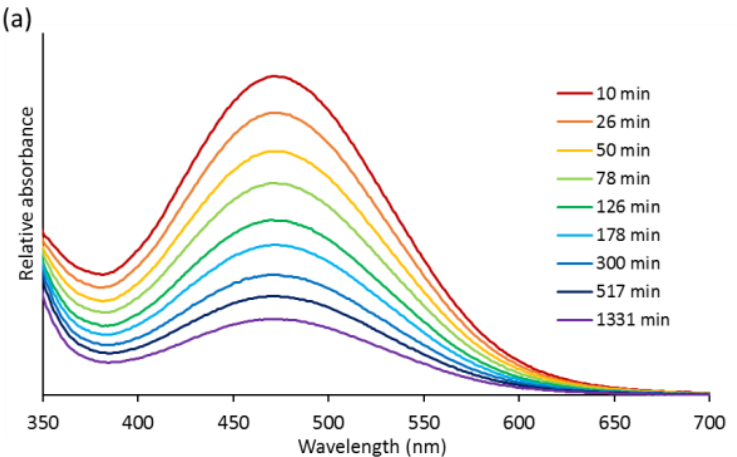

(b)

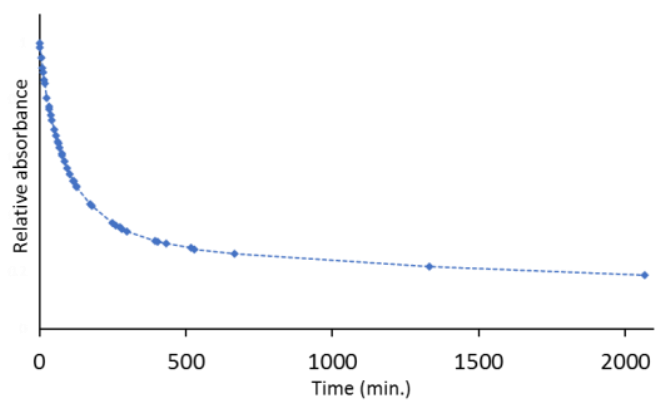

Figure S19. Decay of $\left[\mathbf{2 a}^{\circ+}\right]\left[\mathrm{Cl}^{-}\right]\left(0.16 \mathrm{mM} ; \lambda_{\max }=472 \mathrm{~nm}\right)$ in $0.1 \mathrm{M}$ sulfuric acid. (a) Selected spectra. (b) Absorbance at $472 \mathrm{~nm}$ for 2000 minutes. 


\section{IR Spectroscopy}

\section{General information}

The IR spectra were recorded at room temperature with Cary 600 FTIR Spectrometer (Agilent Technologies) equipped with GladiATR (PIKE technologies).

\section{Experimental spectra}

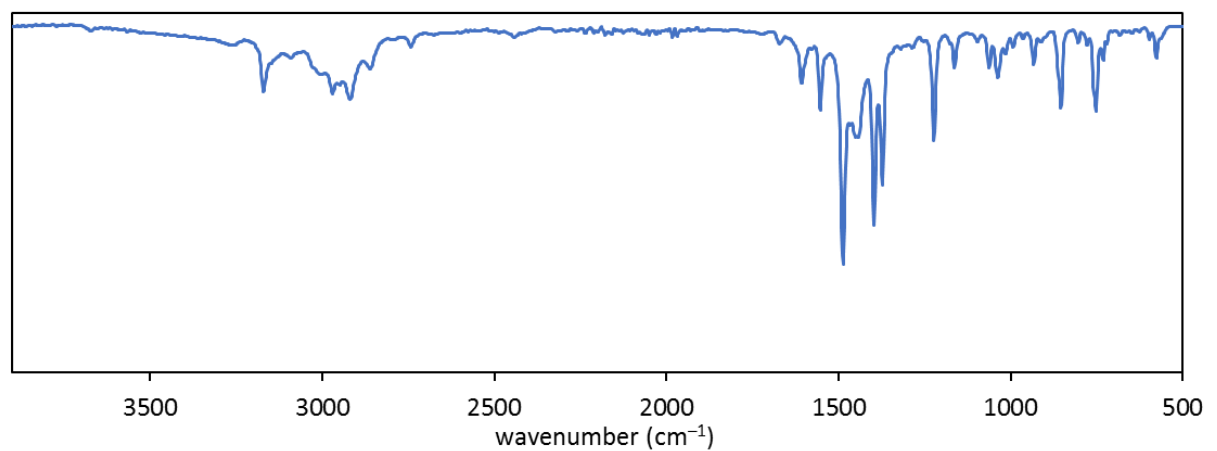

Figure S20. IR spectrum of $\left[2 \mathrm{a}^{\cdot+}\right]\left[\mathrm{Cl}^{-}\right]$.

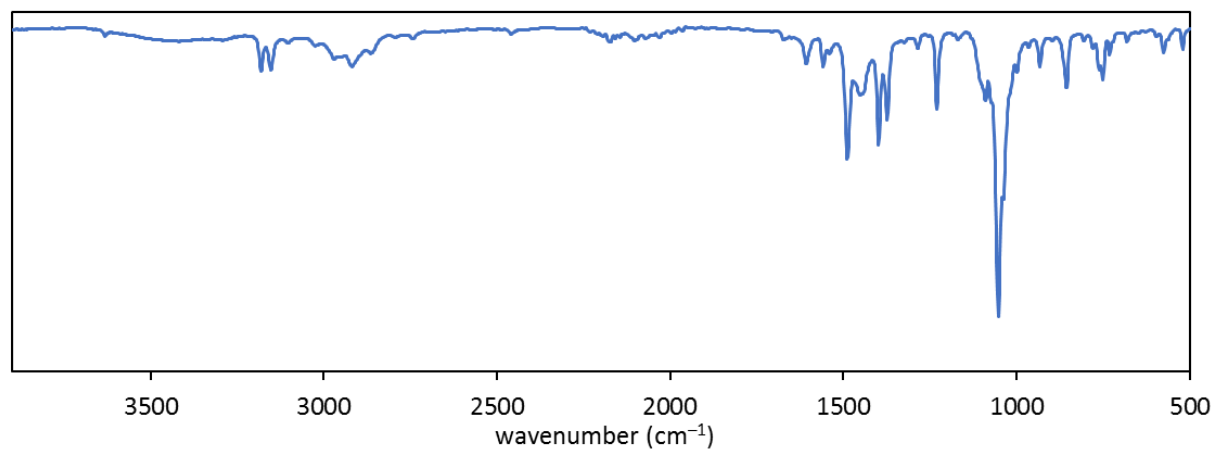

Figure S21. IR spectrum of $\left[\mathbf{2 a}^{\cdot+}\right]\left[\mathrm{BF}_{4}^{-}\right]$.

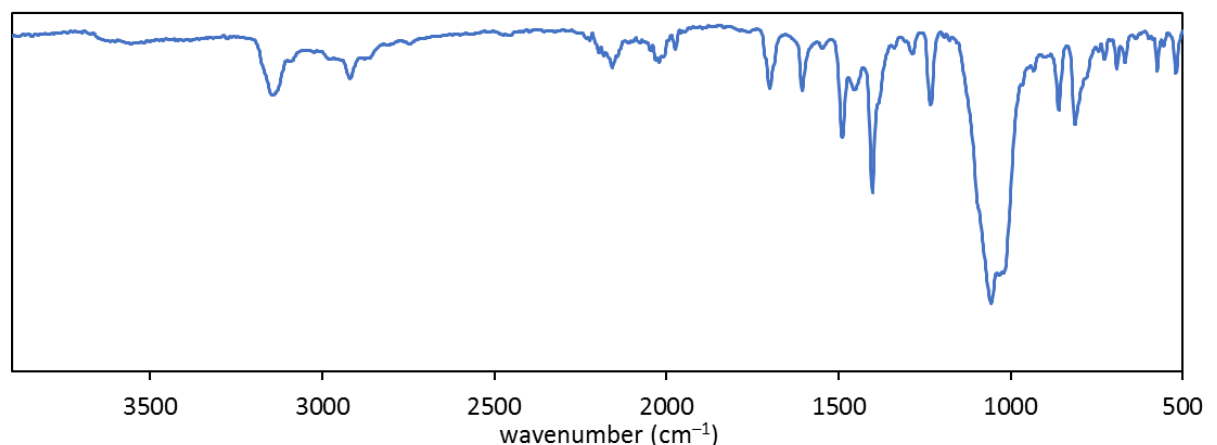

Figure S22. IR spectrum of $\left[\mathbf{2 a}^{2+}\right]\left[\mathbf{B F}_{4}^{-}\right]_{2}$. 


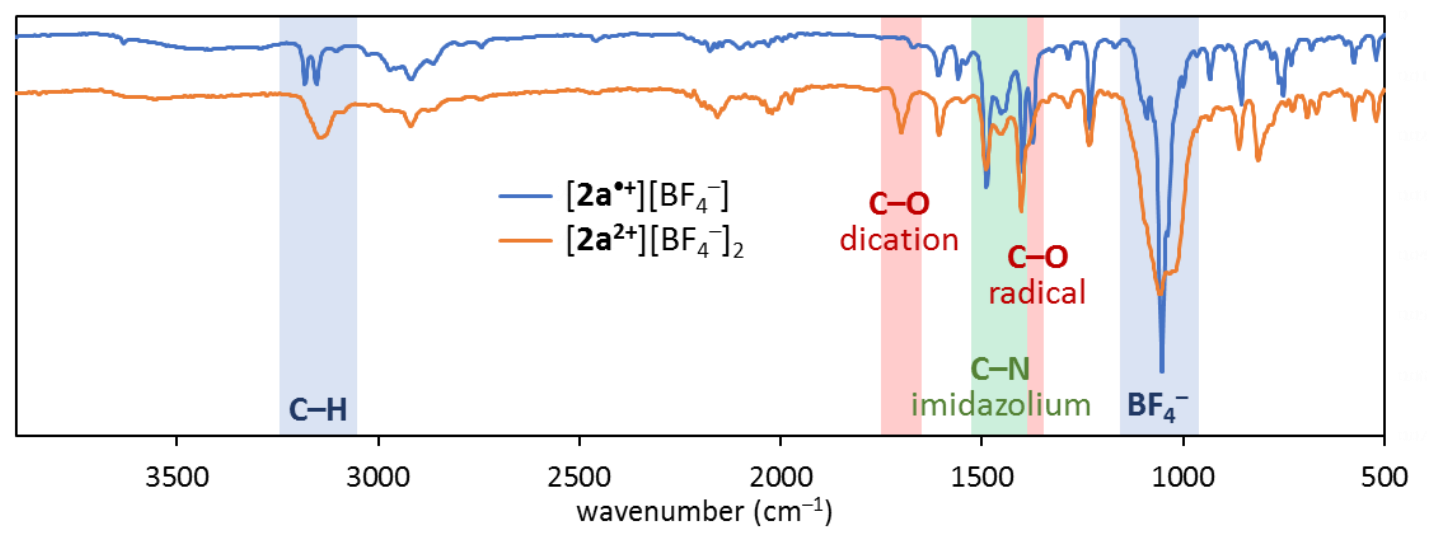

Figure S23. Comparison and signal assignment. 


\section{NMR}

\section{General information}

${ }^{1} \mathrm{H}$ NMR spectra, proton decoupled ${ }^{13} \mathrm{C}$ NMR spectra $\left({ }^{13} \mathrm{C}\left\{{ }^{1} \mathrm{H}\right\}\right)$, and proton decoupled ${ }^{19} \mathrm{~F}$ NMR spectra $\left({ }^{19} \mathrm{~F}\left\{{ }^{1} \mathrm{H}\right\}\right)$ were recorded using either a Bruker DRX 500 spectrometer, Bruker AVANCE III 300 spectrometer, or AVANCE III HD 850 spectrometer. Chemical shifts of ${ }^{1} \mathrm{H}$ and ${ }^{13} \mathrm{C}$ acquisitions were referenced to the residual solvent peaks $\left({ }^{1} \mathrm{H}: \mathrm{CD}_{3} \mathrm{CN}, \delta=1.94 \mathrm{ppm} ; \mathrm{C}_{6} \mathrm{D}_{6}, \delta=7.16 \mathrm{ppm} ;{ }^{13} \mathrm{C}\right.$ : $\left.\mathrm{CD}_{3} \mathrm{CN}, \delta=118.26 \mathrm{ppm} ; \mathrm{C}_{6} \mathrm{D}_{6}, \delta=128.06 \mathrm{ppm}\right) .{ }^{[15]}$

\section{NMR spectra}

${ }^{1} \mathrm{H},{ }^{13} \mathrm{C},{ }^{19} \mathrm{~F}$ NMR spectra of $\left[2 \mathrm{a}^{2+}\right]\left[\mathrm{BF}_{4}^{-}\right]_{2}$

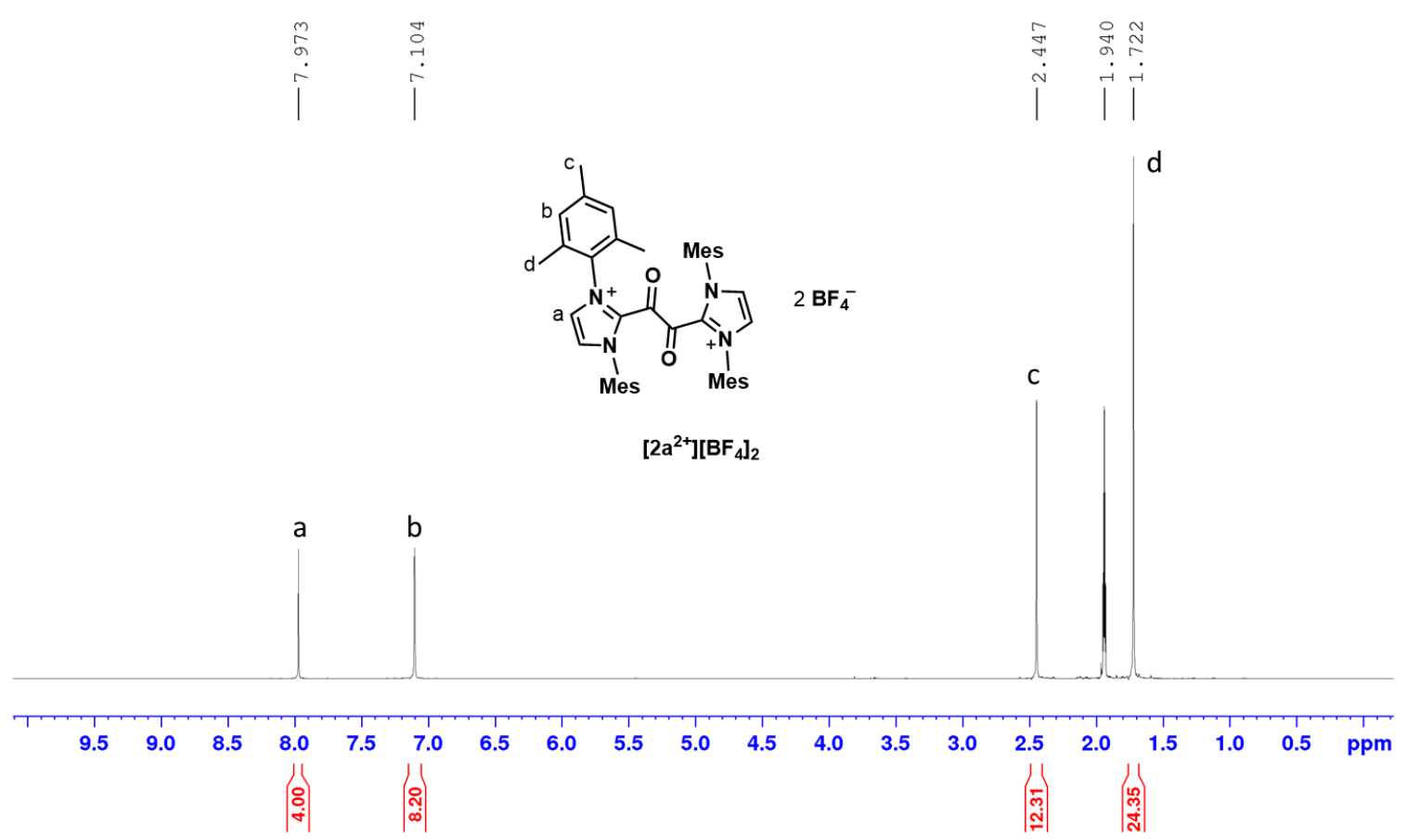

Figure S24. ${ }^{1} \mathrm{H}$ NMR spectrum and assignment of the signals for the compound $\left[\mathbf{2 a}^{2+}\right]\left[\mathbf{B F}_{4}^{-}\right]_{2}$. 


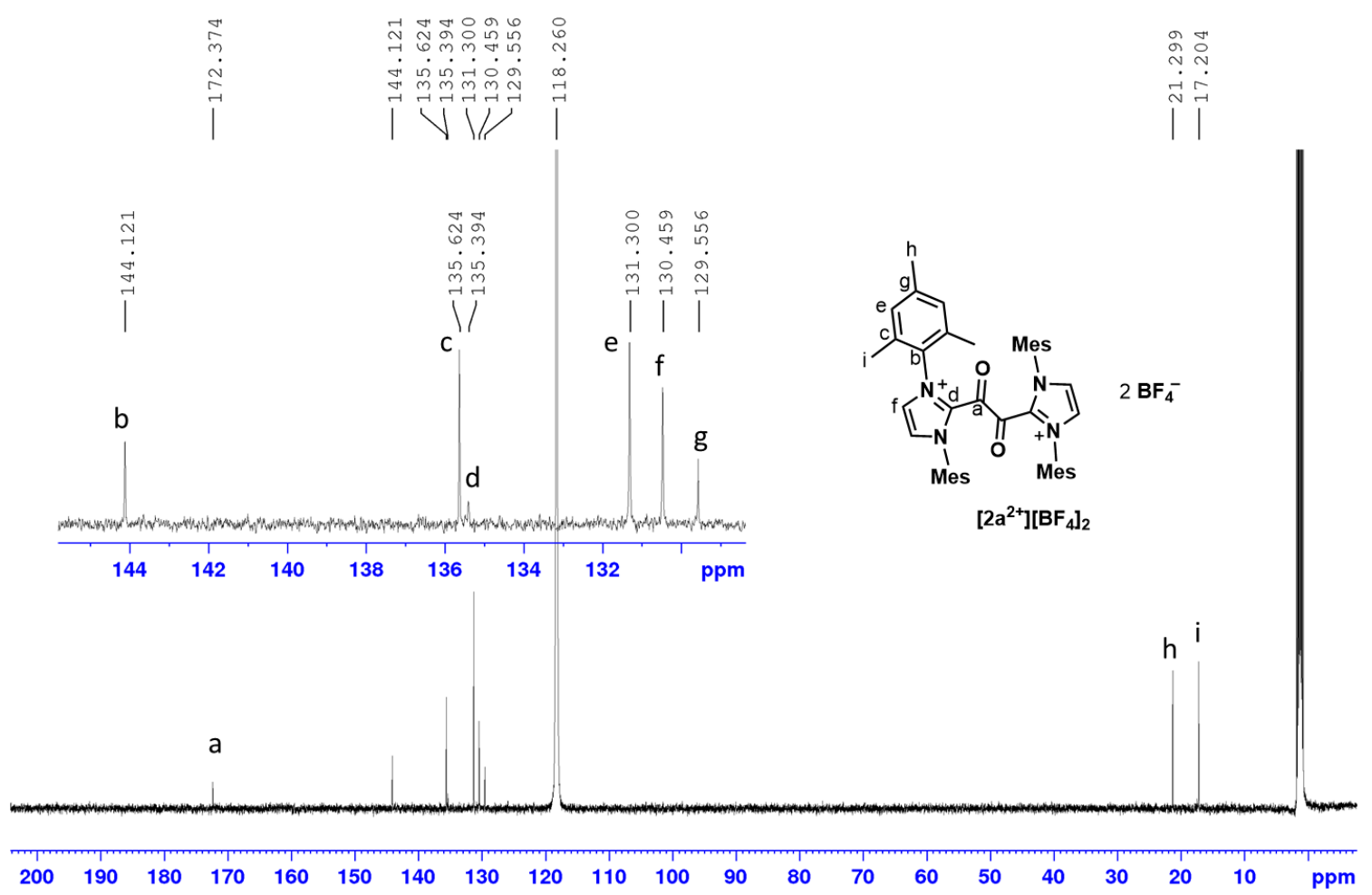

Figure S25. Proton decoupled ${ }^{13} \mathrm{C}$ NMR spectrum and assignment of the signals for the compound $\left[2 \mathrm{a}^{2+}\right]\left[\mathrm{BF}_{4}^{-}\right]_{2}$.

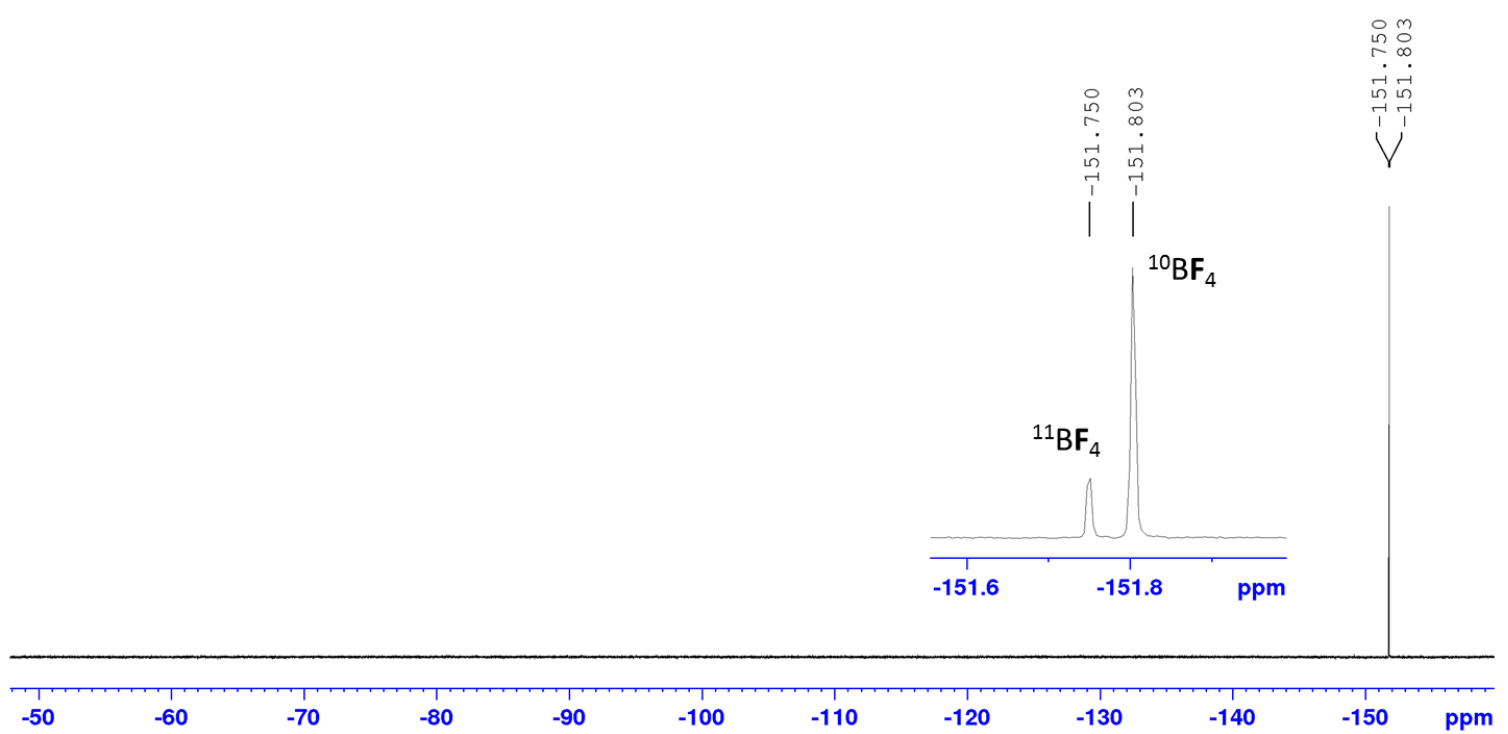

Figure S26. Proton decoupled ${ }^{19} \mathrm{~F}$ NMR spectrum for the compound $\left[\mathbf{2 a}^{2+}\right]\left[\mathbf{B F}_{4}^{-}\right]_{2}$. 
${ }^{1} \mathrm{H},{ }^{13} \mathrm{C}$ NMR spectra of $2 \mathrm{a}^{0}$

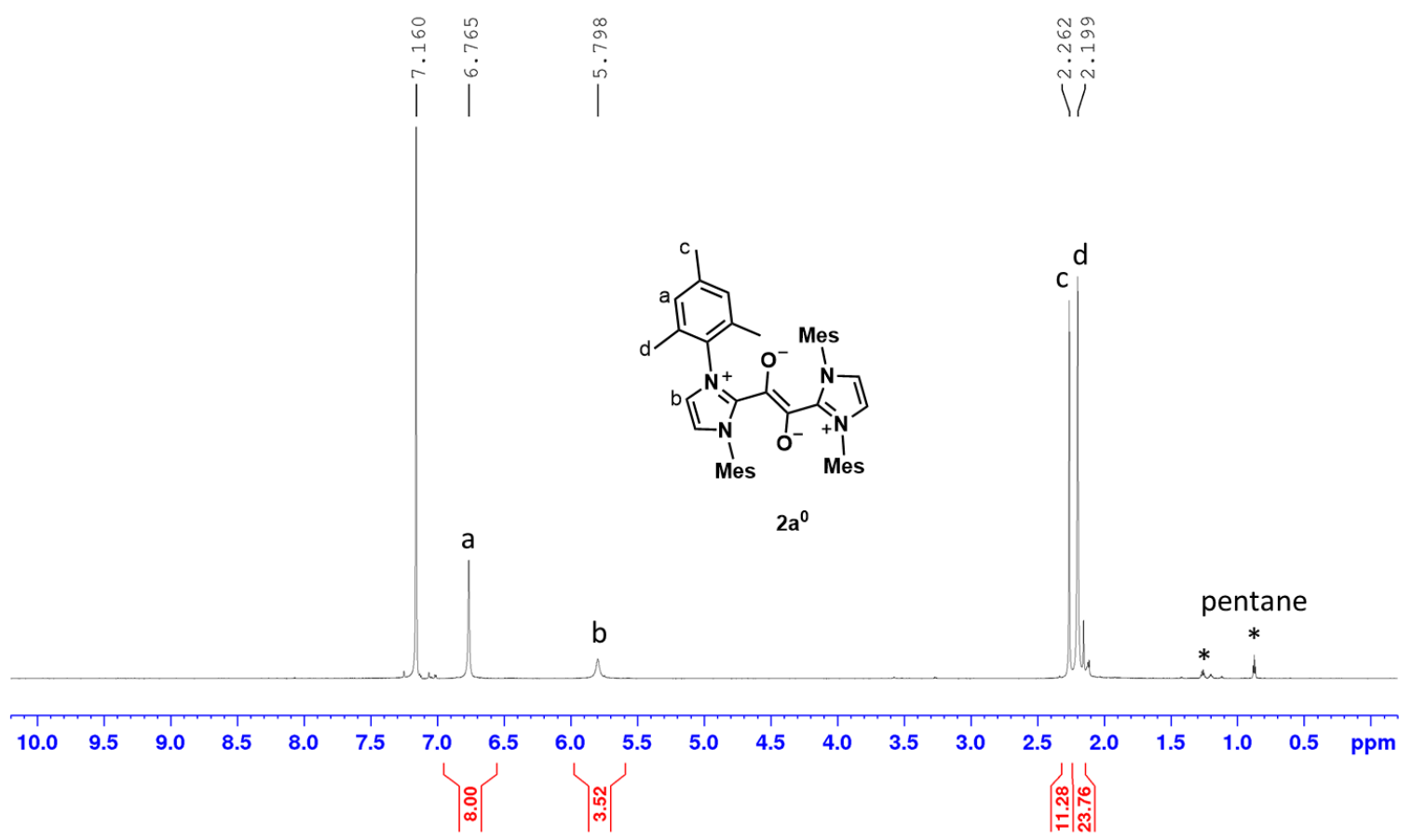

Figure S27. ${ }^{1} \mathrm{H}$ NMR spectrum and assignment of the signals for the compound $\mathbf{2} \mathbf{a}^{\mathbf{0}}$.

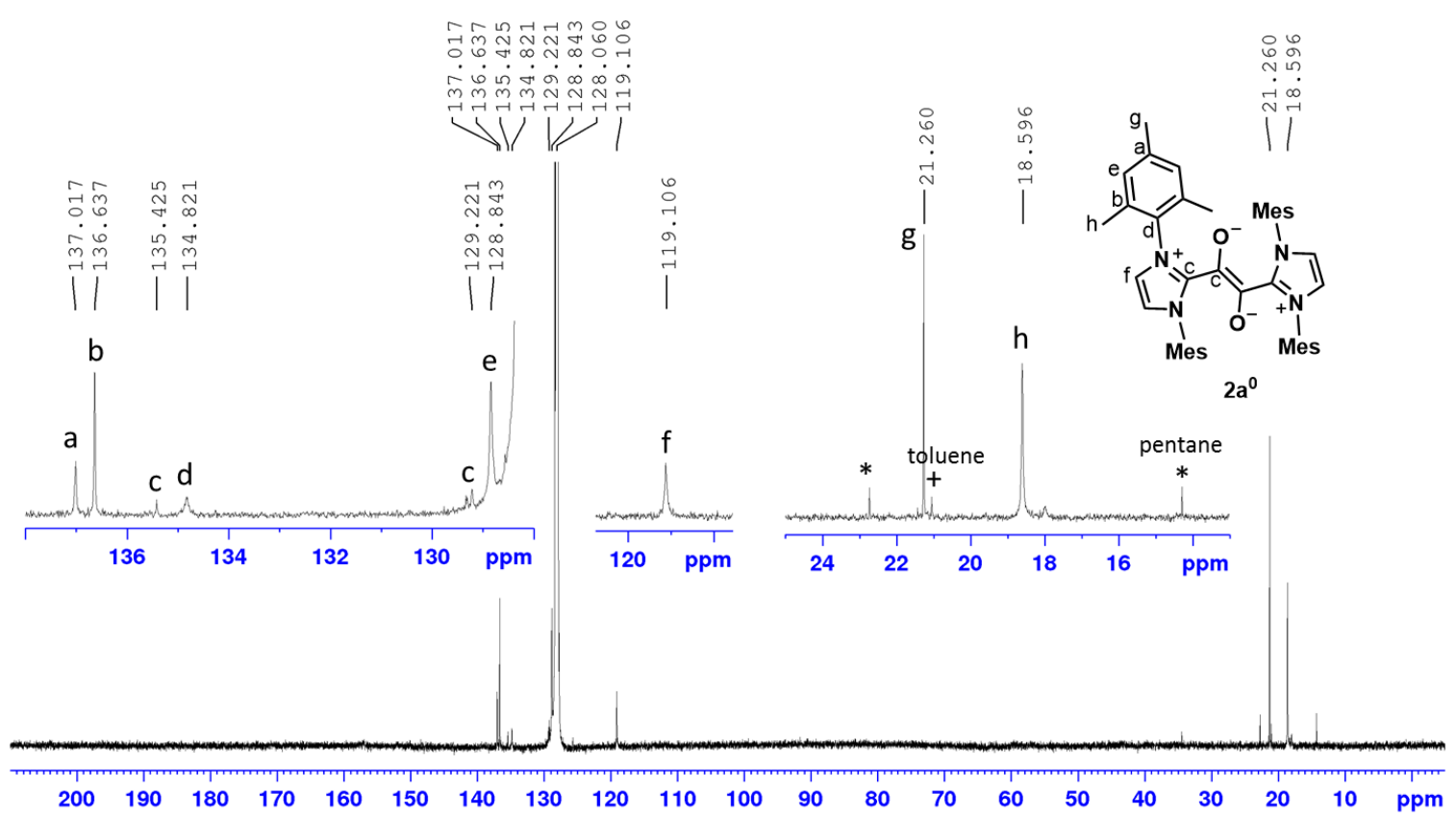

Figure S28. Proton decoupled ${ }^{13} \mathrm{C}$ NMR spectrum and assignment of the signals for the compound $2 \mathbf{a}^{0}$. 
${ }^{1} \mathrm{H},{ }^{19} \mathrm{~F}$ NMR spectra of $2 \mathrm{a}^{{ }^{+}}$

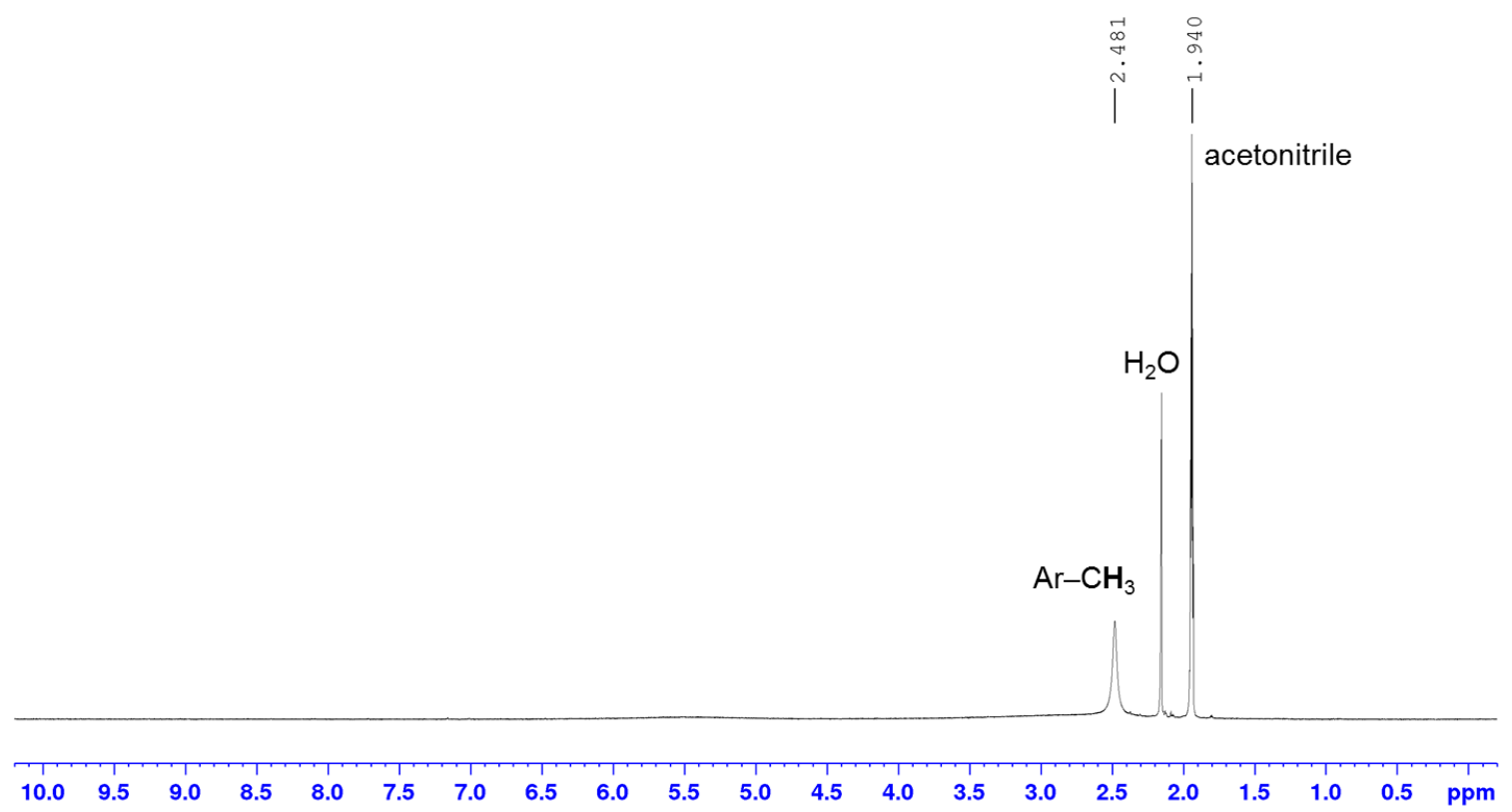

Figure S29. Proton NMR spectrum for the compound $\left[\mathbf{2 a}^{\cdot+}\right]\left[\mathbf{C l}^{-}\right]$.

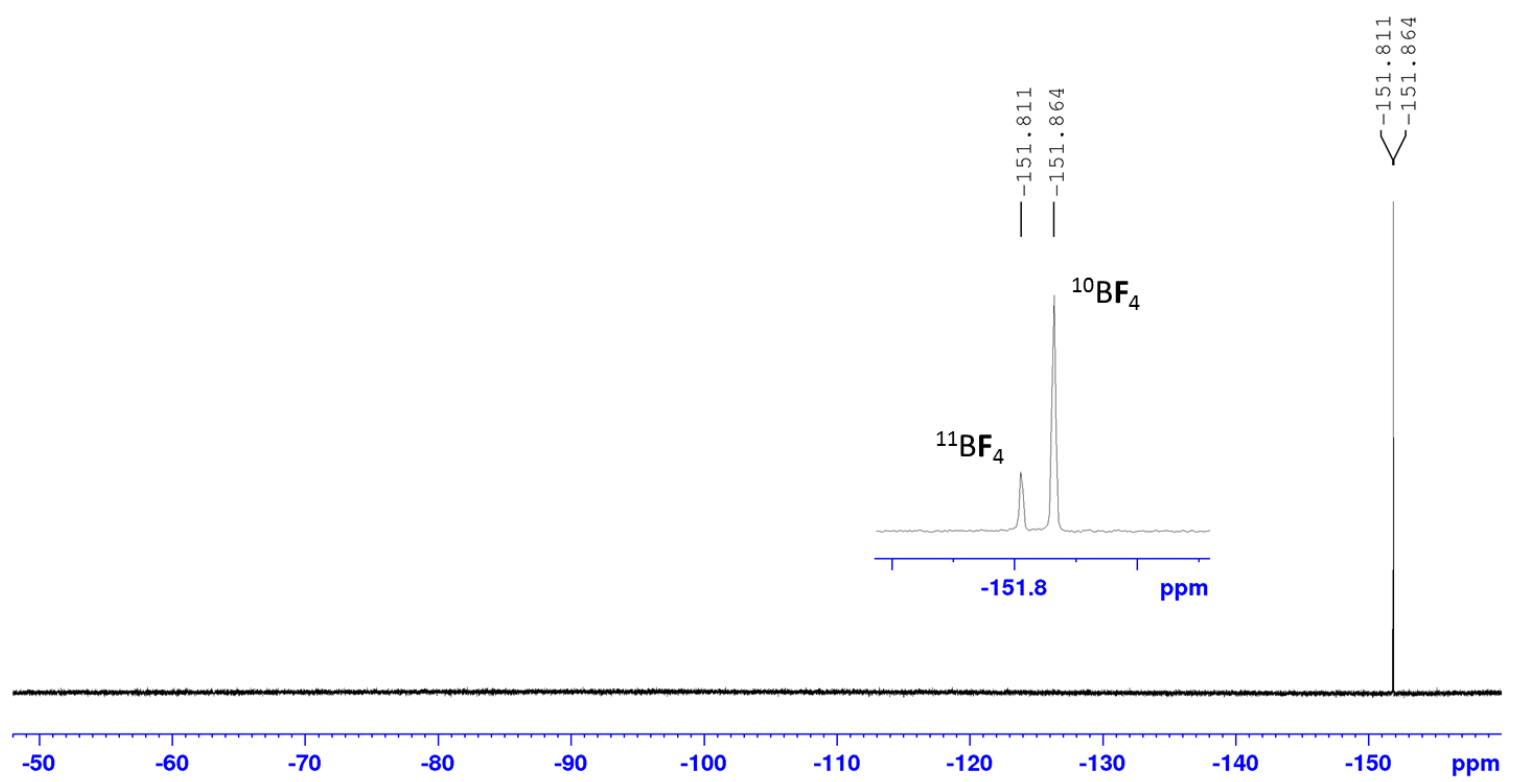

Figure S30. Proton decoupled ${ }^{19} \mathrm{~F}$ NMR spectrum for the compound $\left[\mathbf{2 a}^{\cdot+}\right]\left[\mathbf{B F}_{4}^{-}\right]$. 


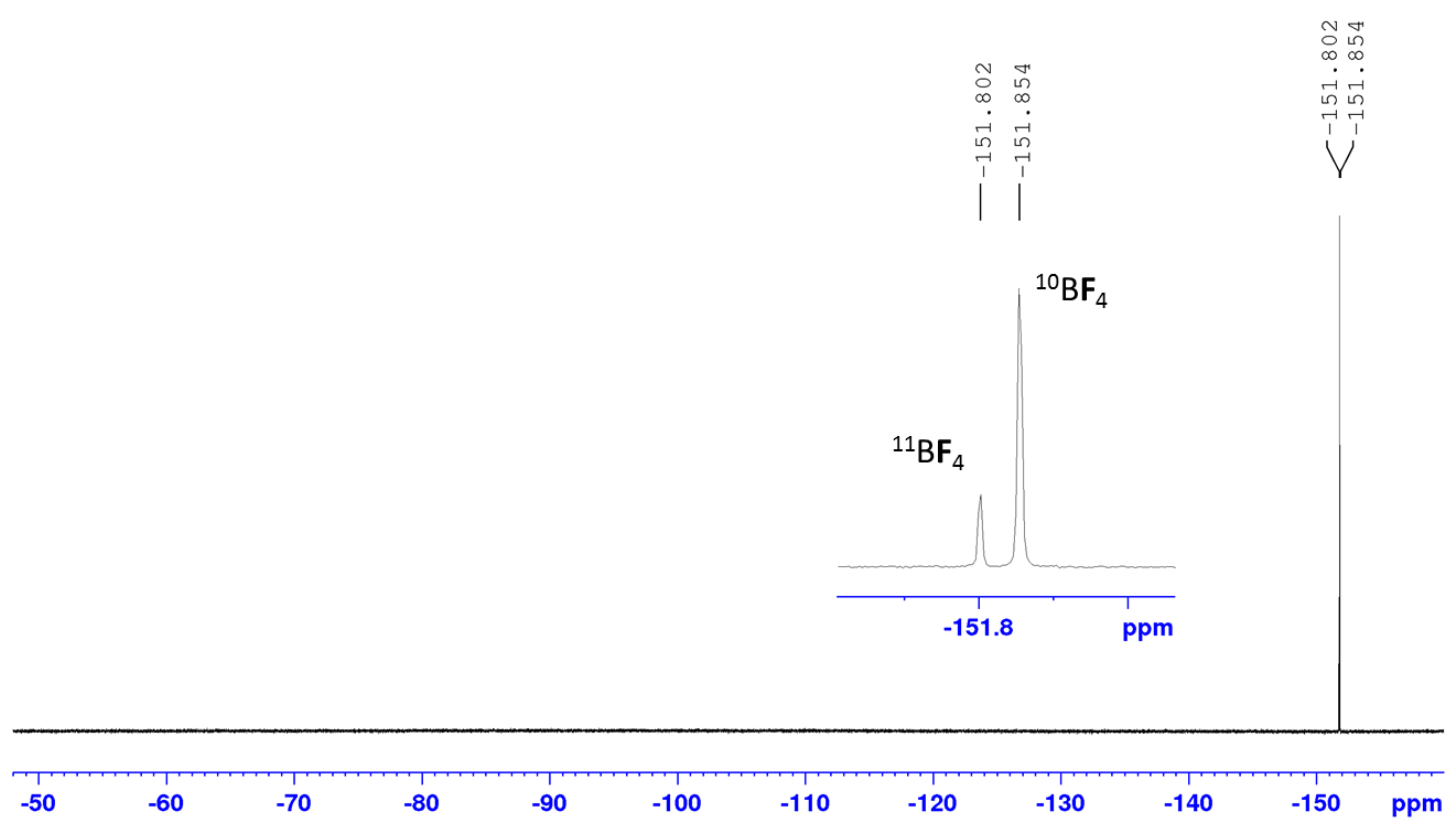

Figure S31. Proton decoupled ${ }^{19} \mathrm{~F}$ NMR spectrum for the compound $\left[\mathbf{2 b}^{\mathbf{}}{ }^{+}\right]\left[\mathbf{B F}_{4}{ }^{-}\right]$. 


\section{References}

[1] X. Bantreil, S. P. Nolan, Nat. Protoc. 2011, 6, 69-77.

[2] Z. Dong, C. Pezzato, A. Sienkiewicz, R. Scopelliti, F. Fadaei-Tirani, K. Severin, Chem. Sci. 2020, $11,7615-7618$.

[3] M. J. Frisch, G. W. Trucks, H. B. Schlegel, G. E. Scuseria, M. A. Robb, J. R. Cheeseman, G. Scalmani, V. Barone, G. A. Petersson, H. Nakatsuji, X. Li, M. Caricato, A. V. Marenich, J. Bloino, B. G. Janesko, R. Gomperts, B. Mennucci, H. P. Hratchian, J. V. Ortiz, A. F. Izmaylov, J. L. Sonnenberg, D. Williams-Young, F. Ding, F. Lipparini, F. Egidi, J. Goings, B. Peng, A. Petrone, T. Henderson, D. Ranasinghe, V. G. Zakrzewski, J. Gao, N. Rega, G. Zheng, W. Liang, M. Hada, M. Ehara, K. Toyota, R. Fukuda, J. Hasegawa, M. Ishida, T. Nakajima, Y. Honda, O. Kitao, H. Nakai, T. Vreven, K. Throssell, J. A. M. Jr., J. E. Peralta, F. Ogliaro, M. J. Bearpark, J. J. Heyd, E. N. Brothers, K. N. Kudin, V. N. Staroverov, T. A. Keith, R. Kobayashi, J. Normand, K. Raghavachari, A. P. Rendell, J. C. Burant, S. S. Iyengar, J. Tomasi, M. Cossi, J. M. Millam, M. Klene, C. Adamo, R. Cammi, J. W. Ochterski, R. L. Martin, K. Morokuma, O. Farkas, J. B. Foresman, D. J. Fox, Gaussian, Inc., Wallingford CT, 2016.

[4] Y. Zhao, D. G. Truhlar, Theor. Chem. Acc. 2007, 120, 215-241.

[5] F. Weigend, R. Ahlrichs, Phys. Chem. Chem. Phys. 2005, 7, 3297-3305.

[6] a) G. A. Petersson, A. Bennett, T. G. Tensfeldt, M. A. Al-Laham, W. A. Shirley, J. Mantzaris, The Journal of Chemical Physics 1988, 89, 2193-2218; b) G. A. Petersson, M. A. Al-Laham, The Journal of Chemical Physics 1991, 94, 6081-6090.

[7] A. V. Marenich, C. J. Cramer, D. G. Truhlar, J. Phys. Chem. B 2009, 113, 6378-6396.

[8] T. Lu, F. Chen, J. Comput. Chem. 2012, 33, 580-592.

[9] a) G. Knizia, J. Chem. Theory Comput. 2013, 9, 4834-4843; b) G. Knizia, J. E. Klein, Angew. Chem. Int. Ed. 2015, 54, 5518-5522.

[10] O. V. Dolomanov, L. J. Bourhis, R. J. Gildea, J. A. K. Howard, H. Puschmann, J. Appl. Crystallogr. 2009, 42, 339-341.

[11] G. M. Sheldrick, Acta. Cryst. 2015, A71, 3-8.

[12] G. M. Sheldrick, Acta. Cryst. 2015, C71, 3-8.

[13] B. Rees, L. Jenner, M. Yusupov, Acta. Cryst. 2005, D61, 1299-1301.

[14] S. Stoll, A. Schweiger, J. Magn. Reson. 2006, 178, 42-55.

[15] G. R. Fulmer, A. J. M. Miller, N. H. Sherden, H. E. Gottlieb, A. Nudelman, B. M. Stoltz, J. E. Bercaw, K. I. Goldberg, Organometallics 2010, 29, 2176-2179. 